\title{
THE GAUSS-BONNET THEOREM FOR RIEMANNIAN POLYHEDRA
}

BY

\author{
CARL B. ALLENDOERFER AND ANDRE WEIL
}

TABLE OF CONTENTS

Section

1. Introduction $\ldots \ldots \ldots \ldots \ldots \ldots \ldots \ldots \ldots \ldots \ldots \ldots \ldots \ldots \ldots \ldots \ldots \ldots$

2. Dual angles in affine space $\ldots \ldots \ldots \ldots \ldots \ldots \ldots \ldots \ldots \ldots \ldots \ldots \ldots \ldots \ldots$

3. Dual angles in Euclidean space. . . . . . . . . . . . . . . . . . . . . . . . 109

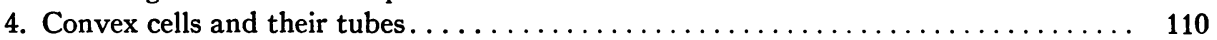

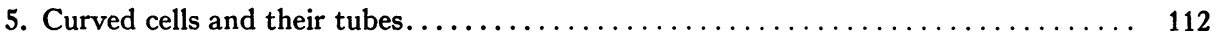

6. Gauss-Bonnet formula for imbedded cells $\ldots \ldots \ldots \ldots \ldots \ldots \ldots \ldots \ldots \ldots \ldots \ldots$

7. Gauss-Bonnet formula for Riemannian polyhedra $\ldots \ldots \ldots \ldots \ldots \ldots \ldots \ldots \ldots \ldots 121$

1. Introduction. The classical Gauss-Bonnet theorem expresses the "curvatura integra," that is, the integral of the Gaussian curvature, of a curved polygon in terms of the angles of the polygon and of the geodesic curvatures of its edges. An important consequence is that the "curvatura integra" of a closed surface (or more generally of a closed two-dimensional Riemannian manifold) is a topological invariant, namely (except for a constant factor) the Euler characteristic.

One of us( $\left.{ }^{1}\right)$ and W. Fenchel( $\left.{ }^{2}\right)$ have independently generalized the latter result to manifolds of higher dimension which can be imbedded in some Euclidean space. For such manifolds, they proved a theorem which we shall show to hold without any restriction, and which may be stated as follows:

THEOREM I. Let $M^{n}$ be a closed Riemannian manifold of dimension $n$, with the Euler-Poincarê characteristic $\chi$; let $d v(z)$ be the Riemannian volume-element at the point with local coordinates $z^{\mu}(1 \leqq \mu \leqq n)$; let $g_{\mu \nu}$ be the metric tensor, $g=\left|g_{\mu \nu}\right|$ its determinant, $R_{\mu_{1} \mu_{2} \nu_{1} \nu_{2}}$ the Riemannian curvature tensor at the same point; and define the invariant scalar $\Psi(z)$ by:

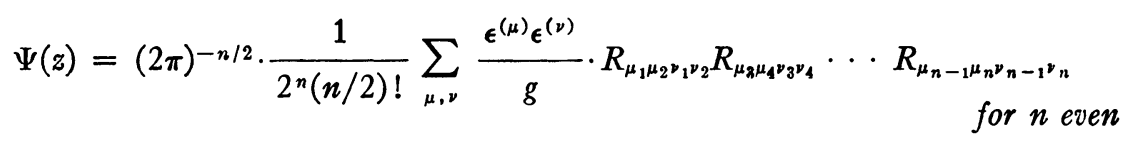

$$
\Psi(z)=0 \text { for } n \text { odd. }
$$

Presented to the Society, December 30, 1941 under the title $A$ general proof of the GaussBonnet theorem; received by the editors April 23, 1942.

(1) C. B. Allendoerfer, The Euler number of a Riemann manifold, Amer. J. Math. vol. 62 (1940) p. 243.

(2) W. Fenchel, On total curvatures of Riemannian manifolds. (I), J. London Math. Soc. vol. 15 (1940) p. 15. The concluding words of this paper show that the author contemplated an extension of his method which was to give him "a formula of Gauss-Bonnet type." We do not know whether such an extension has been published, or even carried out. 
Then:

$$
\chi=\int_{M^{n}} \Psi(z) d v(z)
$$

Here and throughout this paper a sign such as $\sum_{\mu, \nu}$ indicates summation over all indices $\mu_{i}, \nu_{i}$, these indices running independently over their whole range; and $\epsilon^{(\mu)}$ is the relative tensor $\epsilon^{\mu_{1} \mu_{2}} \cdots \mu_{n}$ defined by $\epsilon^{(\mu)}=+1$ if $\left(\mu_{1}, \mu_{2}, \cdots, \mu_{n}\right)$ is an even permutation of $(1,2, \cdots, n), \epsilon^{(\mu)}=-1$ if it is an odd permutation, and $\epsilon^{(\mu)}=0$ otherwise. Owing to the symmetry properties of the curvature tensor it is readily seen that each term in our sum occurs $2^{n}(n / 2)$ ! times or a multiple of that number; for that reason, in our arrangement of the numerical factor, the sign $\Sigma$ is preceded by the inverse of that integer, so that the sum under $\Sigma$, together with the factor immediately in front of it, is (except for $1 / \mathrm{g}$ ) a polynomial in the R's with integer coefficients; similar remarks apply to the other formulae in this paper. On the other hand, it may be convenient, for geometric reasons, to define the curvature as $K=\omega_{n} / 2 \cdot \Psi(z)$, where $\omega_{n}$ is the surface-area of the unit-sphere $S^{n}$ in $R^{n+1}$, so that the curvature is 1 for that sphere if $n$ is even $\left(^{3}\right)$; Theorem I then gives $\int K d v(z)=\omega_{n} \cdot \chi / 2$.

It does not seem to be known at present whether every closed Riemannian manifold can be imbedded in a Euclidean space. However, the possibility of local imbedding, at least in the analytic case, has been proved by E. Car$\tan \left({ }^{4}\right)$, and this naturally suggests applying the same method of tubes, which was developed for closed imbedded manifolds in the above-mentioned paper( $\left.{ }^{1}\right)$, to the cells of a sufficiently fine subdivision of an arbitrary manifold. This gives a theorem on imbedded cells which is the $n$-dimensional analogue of the Gauss-Bonnet formula; the corresponding theorem for polyhedra will emerge as the main result of the present paper; except for details which will be filled in later, this can be stated as follows.

In a Riemannian manifold $M^{n}$, let $M^{r}$ be a differentiable submanifold of dimension $r<n$; we assume that $M^{r}$ is regularly imbedded in $M^{n}$, that is, taking local coordinates $\zeta^{i}$ on $M^{r}$ and $z^{\mu}$ on $M^{n}$, that the matrix $\left\|\partial z^{\mu} / \partial \zeta^{i}\right\|$ is of rank $r$. We introduce the following tensors. First, we write:

$$
\mathrm{P}_{i_{1} i_{2} j_{1} j_{2}}=\sum_{\mu \nu} R_{\mu_{1} \mu_{2} \nu_{1} \nu_{2}} \cdot \frac{\partial z^{\mu_{1}}}{\partial \zeta^{i_{1}}} \cdot \frac{\partial z^{\mu_{2}}}{\partial \zeta^{i_{2}}} \cdot \frac{\partial z^{\nu_{1}}}{\partial \zeta^{j_{1}}} \cdot \frac{\partial z^{\nu_{2}}}{\partial \zeta^{j_{2}}}
$$

(3) It will be noticed that for $n$ even the numerical factor in $1 / 2 \cdot \omega_{n} \cdot \Psi(z)$ as calculated from (1) has, owing to the value of $1 / 2 \cdot \omega_{n}=2^{n / 2} \cdot(2 \pi)^{n / 2} \cdot(n / 2) ! / n !$, a simple rational value.

(4) E. Cartan, Sur la possibilité de plonger un espace riemannien donné dans un espace euclidien, Annales de la Société Polonaise de Mathématique vol. 6 (1927) p. 1. This followed a paper by M. Janet under the same title, ibid. vol. 5 (1926) p. 38, where an incomplete proof of the same theorem is given; Janet's proof was completed by C. Burstin, Ein Beitrag zum Problem der Einbettung der Riemannschen Räume in euklidischen Räumen, Rec. Math. (Mat. Sbornik) N.S. vol. 38 (1931) p. 74. 
those being the components of the curvature tensor of the imbedding manifold $M^{n}$ in the directions which are tangent to $M^{r}$. Next, let $x$ be a normal vector to $M^{r}$ in $M^{n}$, with the covariant components $x_{\mu}$; we write

$$
\Lambda_{i j}(x)=-\sum_{\nu} x_{\nu}\left[\frac{\partial^{2} z^{\nu}}{\partial \zeta^{i} \partial \zeta^{j}}+\sum_{\lambda \mu}\left\{\begin{array}{c}
\nu \\
\lambda \mu
\end{array}\right\} \frac{\partial z^{\lambda}}{\partial \zeta^{i}} \cdot \frac{\partial z^{\mu}}{\partial \zeta^{j}}\right] .
$$

where

$$
\left\{\begin{array}{c}
\nu \\
\lambda \mu
\end{array}\right\}
$$

are the Christoffel symbols in $M^{n}$. The $\Lambda$ 's are linear combinations of the coefficients of the second fundamental form of $M^{r}$ in $M^{n}$. We now introduce, for $0 \leqq 2 f \leqq r$, the combinations

$$
\begin{aligned}
\Phi_{r, f}(\zeta, x)= & \frac{1}{2^{2 f} \cdot f !(r-2 f) !} \sum_{i, j} \frac{\epsilon^{(i)} \cdot \epsilon^{(j)}}{\gamma} \\
& \cdot P_{i_{1} i_{2} j_{1} j_{2}} \cdots \mathbf{P}_{i_{2 f-1} i_{2} j_{2 f-1} j_{2 f}} \cdot \Lambda_{i_{2 f+1} j_{2 f+1}}(x) \cdots \Lambda_{i_{r} j_{r}}(x)
\end{aligned}
$$

where $\gamma$ is the determinant of the metric tensor $\gamma_{i j}$ on $M^{r}$. Let now $S^{n-r-1}$ be the unit-sphere in the normal linear manifold $N^{n-r}(\zeta)$ to $M^{r}$ at $\zeta$; calling $\xi$ an arbitrary point on that sphere, that is, an arbitrary unit-vector $\left({ }^{5}\right)$, normal to $M^{r}$ at $\zeta$, we denote by $d \xi$ the area-element at $\xi$ on $S^{n-r-1}$; and finally, we consider the expression $\left({ }^{6}\right)$

$$
\Psi\left(\zeta, \xi \mid M^{r}\right)=\frac{\pi^{-n / 2} \Gamma(n / 2)}{2} \cdot \sum_{f=0}^{[r / 2]} \frac{\Phi_{r, f}(\zeta, \xi)}{(n-2)(n-4) \cdots(n-2 f)} \cdot d \xi,
$$

which can be integrated over the whole or part of the sphere $S^{n-r-1}$.

Let now $P^{n}$ be a Riemannian polyhedron, that is, a manifold with a boundary, the boundary consisting of polyhedra $P_{\lambda}^{r}$ of lower dimensions (precise definitions will be given in $\S 7) ; z^{\mu}$ and $\zeta^{i}$ being local coordinates in $P^{n}$ and $P_{\lambda}^{r}$, respectively, in the neighborhood of a point $\zeta$ of $P_{\lambda}^{r}$, we consider the set $\Gamma(\zeta)$ of all unit-vectors $\xi$ at that point, with components $\xi_{\mu}$ such that $\sum_{\mu} \xi_{\mu} \cdot d z^{\mu} / d s \leqq 0$ when the derivatives $d z^{\mu} / d s$ are taken along any direction contained in the angle of $P^{n}$ at $\zeta$ (for more details, see $\left.\S \S 6-7\right) . \Gamma(\zeta)$ is found to be a spherical cell, bounded by "great spheres," on the unit-sphere $S^{n-r-1}$ in the normal linear manifold to $P_{\lambda}^{r}$ at $\zeta$, and is what we call the "outer angle" of $P^{n}$ at $\zeta$.

(5) We consistently (except for a short while in the proof of Lemma 8, 87) make no distinction between vectors and their end points, and therefore none between unit-vectors and points on the unit-sphere.

(6) In view of the geometrical nature of the problem, one may suspect that the numerical coefficients in $\Psi$ are connected with areas of spheres; and bringing out such connections may point the way to geometrical interpretations of our formulae. For instance, we have: $\pi^{-n / 2} \cdot \Gamma(n / 2) /\left(2 \cdot 2^{2 f} \cdot f !(r-2 f) !(n-2)(n-4) \cdots(n-2 f)\right)=2 /\left(\omega_{n-2 f-1} \cdot \omega_{2 f} \cdot(2 f) !(r-2 f) ! 2^{f}\right)$. 
Our main theorem, which includes Theorem I as a particular case, expresses in terms of the above quantities the inner characteristic $\chi^{\prime}\left(P^{n}\right)$ of $P^{n}$, that is, the Euler-Poincaré characteristic of the open complex consisting of all inner cells in an arbitrary simplicial or cellular subdivision of $P^{n}$; our methods would enable us to give a similar expression for the ordinary characteristic. The result is as follows:

ThEOREM II. $P^{n}$ being a Riemannian polyhedron, with a boundary consisting of the polyhedra $P_{\lambda}^{r}$, we have:

$$
(-1)^{n} \chi^{\prime}\left(P^{n}\right)=\int_{P^{n}} \Psi(z) d v(z)+\sum_{r=0}^{n-1} \sum_{\lambda} \int_{P_{\lambda}^{r}} d v(\zeta) \int_{\Gamma_{(\zeta)}} \Psi\left(\zeta, \xi \mid P_{\lambda}^{r}\right)
$$

It will be shown in $\$ 6$ how the method of tubes, applied to an imbedded cell in a Euclidean space, leads directly to the formula in Theorem II for such a cell. Sections 2-3 give the necessary details on dual angles and outer angles, and contain the proof of the important additivity property for outer angles in affine space, which is stated in Theorem III; this may be considered as a theorem in spherical geometry, and is a wide generalization of some known results on polyhedra in $R^{3}$; it also includes some results of Poincaré on the angles of Euclidean and spherical polyhedra. Sections 4-5 are mainly devoted to the definition of the tube of a curved cell, and the investigation of its topological properties.

The proof of the main theorem then follows in $\$ 7$, where it is shown how the additivity property for outer angles, proved in $\$ 2$, implies an additivity property for the right-hand side in the formula in Theorem II; hence Theorem II is true for a polyhedron $P^{n}$ if it is true for every polyhedron in a subdivision of $P^{n}$. In particular, it is true for an analytic cell because, by Cartan's theorem, every cell in a sufficiently fine subdivision of such a cell is imbeddable; by an elementary approximation theorem of $\mathrm{H}$. Whitney, it is therefore true for an arbitrary cell. Hence it holds for every polyhedron which can be triangulated into cells; but it is known that every polyhedron can be so triangulated, and this completes the proof. Owing to the very unsatisfactory condition of our present knowledge of differentiable polyhedra, it has been found necessary to include, in $\$ 7$, the proof of some very general lemmas on the subdivisions of such polyhedra; and the section concludes with some remarks about the validity of Theorem II for more general types of polyhedra than those we are dealing with.

2. Dual angles in affine space. It has often been observed that the word "angle" as used in elementary geometry is ambiguous, for it sometimes refers to a subset of the plane bounded by two rays and sometimes to what essentially is a 1-chain on the unit-circle. In order to preserve analogies with elementary geometry, we shall here use the word "angle" both for certain subsets of an affine vector-space $R^{n}$ and for certain $(n-1)$-chains in the mani- 
fold of directions from $O$ in $R^{n}$; this will be done in such a way that no confusion may arise. Even in affine space we shall adopt the unit-sphere $S^{n-1}$, that is, the surface $\sum_{\mu}\left(x^{\mu}\right)^{2}=1$, as a convenient homeomorphic image of the manifold of directions from $O$ in $R^{n}$; in the present section, any other such image could be used just as well to the same purpose.

In this section, $R^{n}$ will denote an affine $n$-dimensional space over the field of real numbers. Assuming that a basis has been chosen in $R^{n}$ once for all, we denote by $x^{\mu}(1 \leqq \mu \leqq n)$ the components of a vector $x$ in $R^{n}$ with respect to that basis. As functions of $x$, the $n$ components $x^{\mu}$ are linear forms in $R^{n}$; and they constitute a basis for the vector-space $\bar{R}^{n}$ of all linear forms $(y, x)=\sum_{\mu} y_{\mu} \cdot x^{\mu}$ over $R^{n}$; the $y_{\mu}$ are then the components, with respect to that basis, of the form $(y, x)$, or, as we may say for short, of the form $y$. We call $\bar{R}^{n}$ the dual space to $R^{n}$. We shall consider linear manifolds $V^{r}$ in $R^{n}$, which, throughout $\$ \S 2-3$, should be understood to contain $O$; throughout this paper, the superscript, when used for a space or manifold, should be understood to indicate the dimension. To every $V^{r}$ in $R^{n}$ corresponds in $\bar{R}^{n}$ the dual manifold $\bar{V}^{n-r}$, consisting of all linear forms which vanish over $V^{r}$ (this should not be confused with the dual space to $V^{r}$ when the latter is considered as an affine space).

Convex angles in $R^{n}$ may be defined in two ways, which may be considered as dual to each other: (a) a convex angle is the set of points $x$ in $R^{n}$ which satisfy a finite number of given inequalities $\left(b_{\nu}, x\right) \geqq 0$; (b) a convex angle is the set of points $x=\sum_{\rho} u_{\rho} \cdot a_{\rho}$, where the $a_{\rho}$ are a finite number of given points, and the numbers $u_{\rho}$ take all values greater than or equal to 0 . It is well known that these two definitions are equivalent. Throughout this paper, all angles will be convex angles, and we shall of ten omit the word "convex."

A convex angle $C$ is said to be of dimension $r$ and of type $s$ if $r$ and $s$ are the dimensions of the smallest linear manifold $V^{r}$ such that $V^{r} \supset C$ and of the largest linear manifold $V^{s}$ such that $C \supset V^{s}$; if $r=s$, the angle reduces to $V^{r}$ and will be called degenerate; otherwise $r>s$. In the notation of angles, the superscript will usually denote the dimension and a Latin subscript the type of the angle whenever it is desirable to indicate either or both. A Greek subscript will be used to distinguish among angles of the same dimension and type.

Let $C$ be an $r$-dimensional angle, contained in the linear manifold $V^{r}$; a point of $C$ will be called an inner point if there is a neighborhood of that point in $V^{r}$ which is contained in $C$; such points form a subset of $C$ which is open with respect to the space $V^{r}$; if $C$ is defined by the inequalities $\left(b_{\nu}, x\right) \geqq 0$, a point $a$ in $C$ will be an inner point if, and only if, all those of the forms $\left(b_{v}, x\right)$ which do not vanish on $V^{r}$ are greater than 0 at $a$. For $r=0, V^{r}$ and $C$ both reduce to the point $O$, which is then considered as an inner point of $C$. The points of an angle which are not inner points constitute a set which is the union of angles of lower dimension; such points are limits of inner points. 
LeMma 1. Let $C$ be a convex angle of dimension $r$, with at least one point a in the open half-space $(b, x)>0$; then its intersection, $D$, with the closed half-space $(b, x) \geqq 0$ is a nondegenerate angle of the same dimension.

For all points of $C$, in a sufficiently small neighborhood of $a$, will be in $D$; among those points there are inner points of $C$, forming an open set in the $V^{r}$ which contains $C$, so that $D$ is $r$-dimensional. Moreover, $D$ contains $a$ and not $-a$, and so cannot be degenerate.

Let $C$ be a convex angle of dimension $m$; a finite set $\mathcal{D}$ of distinct convex angles $C_{\lambda}^{r}\left(0 \leqq r \leqq m ; 1 \leqq \lambda \leqq N_{r}\right)$ will be called a subdivision of $C$ into convex angles whenever the two following conditions are fulfilled: (a) every point of $C$ is an inner point of at least one $C_{\lambda}^{r}$ in $\mathcal{D}$; (b) if two angles $C_{\lambda}^{r}, C_{\mu}^{s}$ in $\mathcal{D}$ are such that there is an inner point of $C_{\lambda}^{r}$ which is contained in $C_{\mu}^{s}$, then $C_{\lambda}^{r} \subset C_{\mu}^{s}$. From (b), it follows that no two distinct angles in $\mathcal{D}$ can have an inner point in common. The angles in $\mathcal{D}$ can be considered, in the usual way, as forming a combinatorial complex. A subdivision of an angle $C$ is called degenerate if it contains a degenerate angle $V^{r}$ of a dimension $r>0$; as $O$ then is an inner point of $V^{r}$ and is in all the angles of $\mathcal{D}$, it follows that all those angles contain $V^{r}$ and are of type at least $r$, as well as $C$ itself. If $\mathcal{D}$ is nondegenerate, it is easily shown to contain angles of all dimensions less than or equal to $m$ and greater than or equal to 0 , and in particular the angle $C^{0}$ which is the point $O$. An angle $C_{\lambda}^{r}$ in $\mathcal{D}$ will be called an inner angle if one of its points is an inner point of $C$; otherwise we call it a boundary angle. All angles $C_{\lambda}^{m}$ of the highest dimension in $\mathcal{D}$ are inner angles.

Let $\left(b_{\iota}, x\right)$ be linear forms in $R^{n}, \iota$ running over a finite set of indices $I$; for every partition of $I$ into three parts $K, L, M$, consider the angle defined by $\left(b_{\kappa}, x\right) \geqq 0(\kappa \in K),\left(b_{\lambda}, x\right) \leqq 0(\lambda \in L),\left(b_{\mu}, x\right)=0(\mu \in M)$; all those angles, or rather those among them which are different from each other, form a subdivision of $R^{n}$. If this process is applied to the set of all linear forms which are needed to define some given angles $C, C^{\prime}, C^{\prime \prime}, \ldots$ in finite number, then the angles of the resulting subdivision which are contained in $C$ form a subdivision of $C$; and the same applies to $C^{\prime}, C^{\prime \prime}, \cdots$.

The intersection of a convex angle of dimension $r \geqq 1$ with the unit-sphere $S^{n-1}$ in $R^{n}$, or, as we shall also say, its trace on $S^{n-1}$, will be called a spherical cell of dimension $r-1$. If the angle is degenerate, so is the cell. A nondegenerate cell is homeomorphic to an "element" (a closed simplex) of the same dimension. A degenerate cell is a sphere.

Let $\Gamma$ be the trace of $C^{m}$ on $S^{n-1}$; let $\Phi$ be a subdivision of $C^{m}$. The traces $\Gamma_{\lambda}^{r-1}$ of the angles $C_{\lambda}^{r}$ of $\mathscr{D}$ on $S^{n-1}$ for $1 \leqq r \leqq m$ form a subdivision of $\Gamma$ into cells, and so, if $\mathscr{D}$ is nondegenerate, into topological elements. We can therefore apply elementary results in combinatorial topology to the calculation of the Euler-Poincaré characteristic of such subdivisions.

Lemma 2. Let $\mathcal{D}$ be a nondegenerate subdivision of the angle $C^{m}$, consisting 
of the angles $C_{\lambda}^{r}\left(0 \leqq r \leqq m ; 1 \leqq \lambda \leqq N_{r}\right)$; let $N_{r}^{\prime}$ be the number of inner angles of dimension $r$ in $\mathcal{D}$; and write:

$$
\chi(\mathcal{D})=\sum_{r=0}^{m}(-1)^{r} \cdot N_{r}, \quad \chi^{\prime}(\mathcal{D})=\sum_{r=0}^{m}(-1)^{r} \cdot N_{r}^{\prime} .
$$

Then, if $C^{m}$ is nondegenerate, we have $\chi(\mathcal{D})=0, \chi^{\prime}(\mathcal{D})=(-1)^{m}$; if $C^{m}$ is degenerate, $\chi(\mathcal{D})=\chi^{\prime}(\mathcal{D})=(-1)^{m}$.

This follows at once from the well known value of the characteristic for elements and for spheres, and from the fact that $N_{0}=1, N_{0}^{\prime}=0$.

Let now $C$ be a convex angle in $R^{n}$, defined as the set of all points $x=\sum_{\rho} u_{\rho} \cdot a_{\rho}$, where the $a_{\rho}$ are given points and the $u_{\rho}$ take all values greater than or equal to 0 . A linear form $(y, x)$ will be less than or equal to 0 on $C$ if, and only if, $\left(y,-a_{\rho}\right) \geqq 0$ for all $\rho$; the set of all points $y$ in $\bar{R}^{n}$ with that property is therefore a convex angle $\bar{C}$. The relationship between $C$ and $\bar{C}$ is easily shown to be reciprocal; we shall say that $C$ and $\bar{C}$ are $d u a l$ to each other. If two angles $C, D$ are such that $C \supset D$, then their dual $\bar{C}, \bar{D}$ are such that $\bar{C} \subset \bar{D}$. If an angle is degenerate and reduces to the linear manifold $V^{r}$, then its dual is the dual manifold $\bar{V}^{n-r}$. It follows that if $V^{r} \supset C \supset V^{s}$, then $\bar{V}^{n-r} \subset \bar{C} \subset \bar{V}^{n-s}$; if, therefore, $C$ is of dimension $r$ and type $s$, its dual $\bar{C}$ is of dimension $n-s$ and type $n-r$.

Lemma 3. Let $\bar{C}$ be the dual of an angle $C$ of type $s$, and $C \supset V^{s}$. $A$ point $b$ of $\bar{C}$ is an inner point of $\bar{C}$ if and only if the form $(b, x)$ is less than 0 at all points of $C$ other than those of $V^{s}$.

Let $C$, as above, be the set of points $x=\sum_{\rho} u_{\rho} \cdot a_{\rho}$ when the $u$ 's take all values greater than or equal to 0 . Then $\bar{C}$ is defined by the inequalities $\left(y,-a_{\rho}\right) \geqq 0$, is of dimension $n-s$, and is contained in the dual $\bar{V}^{n-s}$ to $V^{s}$. We have seen that $b$ is an inner point of $\bar{C}$ if and only if $\left(b,-a_{\rho}\right)>0$ for all those values of $\rho$ for which $\left(y,-a_{\rho}\right)$ does not vanish on $\bar{V}^{n-s}$, that is, for which $a_{\rho}$ does not lie in $V^{s}$; this obviously implies the truth of our lemma.

We now introduce the unit-sphere $\bar{S}^{n-1}$ in $\bar{R}^{n}$ (to which our earlier remarks about spheres apply); and we shall use the subdivisions of $\bar{S}^{n-1}$, induced by the subdivisions of $\bar{R}^{n}$ into convex angles, in order to define chains on $\bar{S}^{n-1}$ in the sense of combinatorial topology. All chains should be understood to be $(n-1)$-chains on $\bar{S}^{n-1}$ built up from such subdivisions, the ring of coefficients being the ring of rational integers. We make the usual identifications between certain chains belonging to different subdivisions, by the following rule: if $\mathcal{D}^{\prime}$ is a refinement of $\mathscr{D}$, and a cell $\Gamma^{n-1}$ of $\mathcal{D}$ is the union of cells $\Delta_{\alpha}^{n-1}$ of $\mathscr{D}^{\prime}$, we put $\Gamma^{n-1}=\sum_{\alpha} \Delta_{\alpha}^{n-1}$. With that convention, any $n$-dimensional angle $\bar{C}$ defines a chain, namely, the cell $\Gamma=\bar{C} \cap \bar{S}^{n-1}$, taken with coefficient +1 in a suitable subdivision. An angle of dimension less than $n$ is considered as defining the chain 0 . Angles being given in $\bar{R}^{n}$ in finite number, there are 
always subdivisions of $\bar{S}^{n-1}$ in which the traces of all those angles appear as chains: we get such a subdivision by making use of all the linear forms which appear in the definition of our angles, as previously explained.

Let $C$ be any convex angle in $R^{n}$, and $\bar{C}$ its dual; the chain defined by $\bar{C}$ on $\bar{S}^{n-1}$ will be called the outer angle belonging to $C$, and will be denoted by $\Omega(C)$; that is the chain consisting of the cell $\bar{C} \cap \bar{S}^{n-1}$ if $\bar{C}$ is of dimension $n$, that is if $C$ is of type 0 ; if $C$ is of type greater than $0, \bar{C}$ is of dimension less than $n$, and $\Omega(C)=0$. With that definition, we have the following theorem:

TheOREM III. In a subdivision $\mathcal{D}$ of a convex angle $C$ of dimension $m$, let $C_{\lambda}^{r}$ $\left(0 \leqq r \leqq m ; 1 \leqq \lambda \leqq N_{r}^{\prime}\right)$ be the inner angles; let $\Omega(C)$ and $\Omega\left(C_{\lambda}^{r}\right)$ be the outer angles belonging to $C$ and to $C_{\lambda}^{r}$, respectively. Then:

$$
\sum_{r=0}^{m} \sum_{\lambda=1}^{N_{r}^{r}}(-1)^{r} \cdot \Omega\left(C_{\lambda}^{r}\right)=(-1)^{m} \cdot \Omega(C) .
$$

We may assume that $\mathcal{D}$ is nondegenerate, as otherwise $C$ and all $C_{\lambda}^{r}$ are of type greater than 0 and $\Omega(C)=\Omega\left(C_{\lambda}^{r}\right)=0$. Let $\Gamma$ be any $(n-1)$-cell in a subdivision of $\bar{S}^{n-1}$ in which $\Omega(C)$ and all $\Omega\left(C_{\lambda}^{r}\right)$ are sums of cells; put $e=1$ or 0 according as $\Gamma$ is contained in $\Omega(C)$ or not, and $e_{r, \lambda}=1$ or 0 according as $\Gamma$ is contained in $\Omega\left(C_{\lambda}^{r}\right)$ or not. We have to prove that $\sum_{r, \lambda}(-1)^{r} \cdot e_{r, \lambda}=(-1)^{m} \cdot e$.

Take first the case $e=1$. Then $\Gamma$ is contained in the dual $\bar{C}$ of $C$, and therefore in the duals of all $C_{\lambda}^{r}$, which all contain $\bar{C}$; all the $e_{r, \lambda}$ are equal to 1 , and our formula reduces to $\sum_{r}(-1)^{r} \cdot N_{r}^{\prime}=(-1)^{m}$, which is contained in Lemma 2.

Take now the case $e=0$. Let $b$ be an inner point of $\Gamma$; call $E$ the angle, or closed half-space, determined by $(b, x) \geqq 0$ in $R^{n}$; call $I$ the subset of $E$ defined by $(b, x)>0$. As $b$ is not in $\bar{C}, C$ has a point in $I$, and therefore (by Lemma 1 ) $D=C \cap E$ is an angle of dimension $m$. Similarly, $C_{\lambda}^{r}$ has a point in $I$ if, and only if, $e_{r, \lambda}=0$, and then $D_{\lambda}^{r}=C_{\lambda}^{r} \cap E$ is a nondegenerate angle of dimension $r$. Every inner point of $D$ is an inner point of $C$, therefore an inner point of a $C_{\lambda}^{r}$; it must be, then, an inner point of the corresponding $D_{\lambda}^{r}$, which shows that those $D_{\lambda}^{r}$ which correspond to values of $r, \lambda$ such that $e_{r, \lambda}=0$ are the inner angles of a subdivision of $D$; if $M_{r}^{\prime}$ is the number of such $D_{\lambda}^{r}$ for a given dimension $r$, we have therefore, by Lemma $2, \sum_{r}(-1)^{r} \cdot M_{r}^{\prime}=(-1)^{m}$; hence, in that case, $\sum_{r} \sum_{\lambda}(-1)^{r} \cdot e_{r, \lambda}=\sum_{r}(-1)^{r} \cdot\left(N_{r}^{\prime}-M_{r}^{\prime}\right)=0$, which completes the proof.

Theorem III applies to angles of any dimension and type, and in particular to degenerate angles. Whenever $C$ is of type greater than $0, \Omega(C)$ is 0 .

We observe here that it is merely in order to simplify our exposition that we do not deal with re-entrant, that is, non-convex angles; all our results apply automatically to such angles, provided Theorem III is used to define the corresponding outer angles; we mean that, $\mathcal{D}$ being a subdivision of a nonconvex angle $C$ into convex angles, $\Omega(C)$ should be defined by the formula in Theorem III; Theorem III may then be used to show that this $\Omega(C)$ does not 
depend upon the choice of $\mathscr{D}$. Even self-overlapping angles could be treated in the same way.

3. Dual angles in Euclidean space. In view of the use to be made of dual angles in $\$ \S 5-7$, we add some remarks on the few circumstances which are peculiar to the case of Euclidean spaces. We therefore assume that a positivedefinite quadratic form $\sum_{\mu \nu} g_{\mu \nu} \cdot x^{\mu} x^{\nu}$, with constant coefficients $g_{\mu \nu}$, is given in the space $R^{n}$ of $\$ 2$. As usual, this is used primarily in order to identify $R^{n}$ with the dual space $\bar{R}^{n}$ by means of the formulae $y_{\mu}=\sum_{\nu} g_{\mu \nu} \cdot x^{\nu}$, or, calling $\left\|g^{\mu \nu}\right\|$ the inverse matrix to $\left\|g_{\mu \nu}\right\|, x^{\mu}=\sum_{\nu} g^{\mu \nu} ; y_{\nu}$; the two spaces being thus identified, $x^{\mu}$ and $y_{\mu}$ are called the contravariant and the covariant components, respectively, of the vector which they define; they are the same when, and only when, cartesian coordinates are chosen in $R^{n}$. We have $\left(x, x^{\prime}\right)=\sum_{\mu \nu} g_{\mu \nu} \cdot x^{\mu} x^{\prime \nu}$; two vectors are called orthogonal if $\left(x, x^{\prime}\right)=0$. The unitsphere $S^{n-1}=\bar{S}^{n-1}$ in $R^{n}$ is then naturally taken to be the set of all unitvectors defined by $(x, x)=1$; only in cartesian coordinates does it appear as $\sum_{\mu}\left(x^{\mu}\right)^{2}=1$. The dual manifold $\bar{V}^{n-r}$ to a given linear manifold $V^{r}$ is now the orthogonal or normal manifold to $V^{r}$, consisting of all vectors which are orthogonal to every vector in $V^{r}$.

Every linear manifold $V^{r}$ may now itself be regarded as a Euclidean space, and identified with the dual space; if $C$ is an angle in $V^{r}$, we may therefore consider its dual taken within $V^{r}$, which will be an angle in $V^{r}$, as well as its dual in $R^{n}$. When applied to an angle of given dimension and type, this leads to the following results, which we state in the notation best suited to later applications.

Let $R^{N}$ be a Euclidean space; let $A_{r}$ be an angle of dimension $n$ and type $r$ in $R^{N}$, contained in the linear manifold $T^{n}$ and containing the linear manifold $T^{r}$; put $q=N-n$, call $N^{q}$ the orthogonal manifold to $T^{n}$, and $N^{n-r}$ the orthogonal manifold to $T^{r}$ within $T^{n}$ : the orthogonal manifold to $T^{r}$ in $R^{N}$ is then the direct sum $N^{n-r}+N^{q}$, consisting of all sums of a vector in $N^{n-r}$ and a vector in $N^{q}$.

If wé take cartesian coordinates $w^{\alpha}(1 \leqq \alpha \leqq N)$ so that the $r$ first basisvectors are in $T^{r}$, the $n-r$ next ones in $N^{n-r}$, and the $q$ last ones in $N^{q}$, the angle $A_{r}$ can be defined by $w^{n+\rho}=0(1 \leqq \rho \leqq q)$ and by a finite number of inequalities of the form $\sum_{\sigma=1}^{n-r} b_{\sigma} \cdot w^{r+\sigma} \geqq 0$. It is then readily seen that the dual $\bar{A}^{N-r}$ of $A_{r}$ in $R^{N}$, and its dual $\bar{A}^{n-r}$ taken within $T^{n}$, are related by the formula: $\bar{A}^{N-r}=\bar{A}^{n-r}+N^{q}$, which means that $\bar{A}^{N-r}$ consists of all sums of a vector in $\bar{A}^{n-r}$ and a vector in $N^{q}$; in other words, a vector is in $\bar{A}^{N-r}$ if and only if its orthogonal projection on $T^{n}$ belongs to $\bar{A}^{n-r}$. Moreover, $\bar{A}^{n-r}$ is the same as the dual, taken within $N^{n-r}$, of the trace of $A_{r}$ on $N^{n-r}$, that trace being an angle of dimension $n-r$ and of type 0 . In this way, questions concerning the dual of an angle of arbitrary dimension and type may be reduced to similar questions concerning the dual of an angle of type 0 and of the highest dimension in a suitable space. The same, of course, could be done in an affine space if desired. 
4. Convex cells and their tubes $\left({ }^{7}\right)$. We consider an affine space $R^{N}$, and its dual $\bar{R}^{N}$. The linear manifolds which we shall now introduce do not necessarily contain $O$.

A convex cell in $R^{N}$ is a compact set of points defined by a finite number of inequalities $\left(b_{v}, z\right) \geqq d_{\nu}$. It is said to be of dimension $n$ if $n$ is the dimension of the smallest linear manifold $W^{n}$ containing it; it is then known to be homeomorphic to an $n$-dimensional element. $K^{n}$ being an $n$-dimensional cell, contained in the linear manifold $W^{n}$, an inner point of $K^{n}$ is a point, a neighborhood of which in $W^{n}$ is contained in $K^{n}$. Inner points of $K^{n}$ form an open set in $W^{n}$; the closure of that set is $K^{n}$, and its complement in $K^{n}$, that is, the boundary of $K^{n}$, consists of a finite number of convex cells $K_{\lambda}^{r}$, where $r$ takes all values greater than or equal 0 and less than or equal to $n-1$. We shall count $K^{n}$ as one of the $K_{\lambda}^{r}$; with that convention, the $K_{\lambda}^{r}$, for $0 \leqq r \leqq n$, form a combinatorial complex of dimension $n . K_{\lambda}^{r}$ is a convex cell in a linear manifold $W_{\lambda}^{r}$; the inner points of $K_{\lambda}^{r}$ are those which belong to no $K_{\mu}^{s}$ for $s<r$. Every point in $K^{n}$ is an inner point of one $K_{\lambda}^{r}$ and one only; and, if an inner point of $K_{\lambda}^{r}$ belongs to $K_{\mu}^{s}$, then $K_{\lambda}^{r} \subset K_{\mu}^{s}$.

$z$ being a point in $K^{n}$, the points $x=\xi \cdot\left(z^{\prime}-z\right)$, where $z^{\prime}$ describes $K^{n}$ and $\xi$ takes all values greater than or equal to 0 , form a convex angle, which can be defined by some of the inequalities $\left(b_{\nu}, x\right) \geqq 0$; this will be called the angle of $K^{n}$ at $z$; conversely, if $x$ is any point in that angle, $z+\epsilon \cdot x$ will be in $K^{n}$ for all sufficiently small $\epsilon \geqq 0$. The angle of $K^{n}$ at $z$ is of dimension $n$, and contained in the linear manifold $V^{n}$, the parallel manifold to $W^{n}$ through $O$; if $z$ is an inner point of $K_{\lambda}^{r}$, the angle of $K^{n}$ at $z$ is of type $r$ and contains $V_{\lambda}^{r}$, the parallel manifold to $W_{\lambda}^{r}$ through $O$; it depends only upon $r$ and $\lambda$, and will be denoted by $C_{r, \lambda}$; its dual $\bar{C}_{\lambda}^{N-r}$ is of dimension $N-r$ and type $N-n$.

LEMMA 4. Let $v$ be a vector in $\bar{R}^{N} ; v$ is in $\bar{C}_{\lambda}^{N-r}$ if, and only if, there is a real number e such that $(v, z)=e$ on $K_{\lambda}^{\top}$ and $(v, z) \leqq e$ on $K^{n} ; v$ is an inner point of $\bar{C}_{\lambda}^{N-r}$ if, and only if, there is an e such that $(v, z)=e$ on $K_{\lambda}^{r}$ and $(v, z)<e$ for all $z$ in $K^{n}$ except those in $K_{\lambda}^{r}$.

As to the first point, let $v$ be in $\bar{C}_{\lambda}^{N-r}$; let $z_{0}$ be in $K_{\lambda}^{r}$; put $e_{0}=\left(v, z_{0}\right)$. For every $z$ in $K^{n}, z-z_{0}$ is in $C_{r, \lambda}$, therefore $\left(v, z-z_{0}\right) \leqq 0$, hence $(v, z) \leqq e_{0}$; therefore $e_{0}$ is the least upper bound of $(v, z)$ on $K^{n}$ and cannot depend upon the choice of $z_{0}$ in $K_{\lambda}^{r}$, so that $(v, z)=e_{0}$ for all $z$ in $K_{\lambda}^{r}$; this proves the first point. Conversely, suppose that $(v, z)=e$ for one $z$ in $K_{\lambda}^{r}$, and that $\left(v, z^{\prime}\right) \leqq e$ for all $z^{\prime}$

(7) Tubes of convex bodies and of surfaces are of course nothing new, being closely related to the familiar topic of parallel curves and surfaces. On some aspects of this topic which belong to elementary geometry, the reader may consult W. Blaschke, Vorlesungen ïber Integralgeometrie. II, Hamburger Mathematische Einzelschrift, no. 22, Teubner, Leipzig and Berlin, 1937 , in particular $\$ 37$; on p. 93 of that booklet, he will find careful drawings of the tube of a triangle in the plane, and of a tetrahedron in 3-space. The volume of the tube of a closed manifold was recently calculated by $\mathrm{H}$. Weyl, On the volume of tubes, Amer. J. Math. vol. 61 (1939) p. 461 ; part of H. Weyl's calculations will be used in our $\$ 6$. 
in $K^{n}$; we have $\left(v, z^{\prime}-z\right) \leqq 0$ for all $z^{\prime}$ in $K^{n}$; this gives $(v, x) \leqq 0$ for all $x$ in $C_{r, \lambda}$, and so $v$ is in $\bar{C}_{\lambda}^{N-r}$. The second part can now easily be deduced from Lemma 3.

$K^{n}$ being compact, every linear form $(v, z)$ has on $K^{n}$ a least upper bound $e$; the intersection of $K^{n}$ with the linear manifold $(v, z)=e$ is then one of the cells $K_{\lambda}^{\top}$. This fact, combined with Lemma 4 , shows that the angles $\bar{C}_{\lambda}^{N-r}$ constitute a subdivision of $\bar{R}^{N}$, according to our definition in $\$ 2$. The angle $C_{n}$ of $K^{n}$ at every inner point is degenerate, and reduces to $V^{n}$; its dual $\bar{C}^{N-n}$ is therefore the dual manifold $\bar{V}^{N-n}$ to $V^{n}$; the subdivision of $\bar{R}^{N}$ which consists of the $\bar{C}_{\lambda}^{N-r}$ is therefore nondegenerate if $N=n$, and degenerate if $N>n$. We leave it as an exercise to the reader to verify that, conversely, every subdivision of $R^{N}$ into convex angles can be thus derived from a convex cell, or rather from a class of convex cells, in $R^{N}$. We observe incidentally that Theorem IJI of $\$ 2$ could now be applied; taking $N=n$, which is the only significant case, the $\Omega\left(\bar{C}_{\lambda}^{n-r}\right)$ are now the spherical cells determined by the $C_{r, \lambda}$ on the unitsphere $S^{n-1}$. In particular, assuming that we are in a Euclidean space, and calling $\mu\left(C_{r, \lambda}\right)$ the spherical measure of the cell determined by $C_{r, \lambda}$ (which is nothing else than the measure of the "solid angle" $\left.C_{r, \lambda}\right)$, we find that $\sum_{r=0}^{n} \sum_{\lambda}(-1)^{r} \cdot \mu\left(C_{r, \lambda}\right)=0$; this is the main result on Euclidean polyhedra in $\mathrm{H}$. Poincaré's paper ${ }^{(8)}$ on polyhedra in spaces of constant curvature; his results on spherical polyhedra could also be derived by similar methods.

Now we take $R^{N}$ as a Euclidean space, distance and scalar product being defined by means of a fundamental quadratic form $(y, y)$; and we consequently identify $\bar{R}^{N}$ with $R^{N}$, as we did in $\S 3$. Let $y$ be any point in $R^{N}$; its set-theoretical distance $\delta(y)$ to $K^{n}$ is a continuous function of $y$. Let $z=z(y)$ be the nearest point to $y$ in $K^{n}$; as $K^{n}$ is a compact convex set, $z(y)$ is uniquely defined and depends continuously upon $y$; the vector $v=y-z(y)$, which is of length $\delta(y)$, therefore also depends continuously upon $y$. That being so, we have $\left(y-z^{\prime}, y-z^{\prime}\right) \geqq(v, v)$ for every $z^{\prime}$ in $K^{n}$. Let $x$ be a vector in the angle of $K^{n}$ at $z ; z^{\prime}=z+\epsilon \cdot x$ is in $K^{n}$ for sufficiently small $\epsilon \geqq 0$, and then $y-z^{\prime}=v-\epsilon \cdot x$, - so that, for small $\epsilon$, we have $(v-\epsilon \cdot x, v-\epsilon \cdot x) \geqq(v, v)$. That implies that $(v, x) \leqq 0$. If, therefore, $z$ is an inner point of $K_{\lambda}^{r}$, so that the angle at $z$ is $C_{r, \lambda}$, $v$ is in $\bar{C}_{\lambda}^{N-r}$. Conversely, let $v$ be in $\bar{C}_{\lambda}^{N-r}$, and $z$ be an inner point of $K_{\lambda}^{r}$; as $z^{\prime}-z$ is in $C_{r, \lambda}$ for every $z^{\prime}$ in $K^{n}$, the same calculation will show that $z$ is the point in $K^{n}$ nearest to $z+v$.

We now consider the set $\Theta^{N}$ of all points $y$ in $R^{N}$ whose distance $\delta(y)$ to $K^{n}$ is at most 1 , and we call it the Euclidean tube of $K^{n}$ in $R^{N}$. As $\Theta^{N}$ is a compact convex set and contains an open set in $R^{N}$, it is homeomorphic to an $N$-dimensional closed element. On the other hand, let $B^{N}$ be the set of all vectors $v$ in $R^{N}$ such that $(v, v) \leqq 1$, the boundary of which is the unit-sphere $S^{N-1}$; let $\tau\left(K^{n}\right)$ be the subset of the direct product $K^{n} \times B^{N}$, consisting of all

(8) H. Poincaré, Sur la généralisation d'un théorème élémentaire de géométrie, C. R. Acad. Sci. Paris vol. 140 (1905) p. 113. 
elements $(z, v)$ of that product such that, if $z$ is an inner point of $K_{\lambda}^{r}, v$ is in $\bar{C}_{\lambda}^{N-\tau}$. We have shown that the relation $y=z+v$ defines a one-to-one bicontinuous correspondence between $\Theta^{N}$ and $\mathcal{T}\left(K^{n}\right)$; the latter, therefore, is a closed subset of $K^{n} \times B^{N}$, homeomorphic to $B^{N}$; by means of the correspondence defined by $y=z+v$, we identify once for all $\Theta^{N}$ and $\tau\left(K^{n}\right)$. Calling $(z(y), v(y))$ the point in $\tau\left(K^{n}\right)$ which is thus identified with $y$ in $\Theta^{N}$, we see that the boundary of $\Theta^{N}$ consists of all points $y$ for which $v(y)$ is on $S^{N-1}$; in other words, the mapping $y \rightarrow v(y)$ of the tube into $B^{N}$ maps the boundary into the boundary. As every $v$ is in at least one $\bar{C}_{\lambda}^{N-r}$, the image of the tube by the mapping $v(y)$ covers the whole of $B^{N}$. If we consider a vertex $z_{0}=K_{\rho}^{0}$ of $K^{n}$, and take for $v_{0}$ an inner point of the angle $\bar{C}_{\rho}^{N}$, all vectors $v$ sufficiently near to $v_{0}$ in $R^{N}$ belong to $\bar{C}_{\rho}^{N}$ and to no other angle $\bar{C}_{\lambda}^{N-r}$, as $\bar{C}_{\rho}^{N}$ is an angle of the highest dimension in the subdivision of $R^{N}$ which consists of the $\bar{C}_{\lambda}^{N-r}$. Every such vector $v$, therefore, is the image, by $v(y)$, of the point $y=z_{0}+v$ and of no other point of $\Theta^{N}$. This shows that in the neighborhood of such a $v_{0}$ the mapping $v(y)$ has the local degree +1 , and so, as it maps boundary into boundary, it has the degree +1 everywhere, provided of course that both $\Theta^{N}$ and $B^{N}$ are given the orientation induced by that of $R^{N}$.

5. Curved cells and their tubes. From now onwards, $K^{n}$ will be a convex cell in an affine space $R^{n}$; the object of $\$ \$ 5-6$ will be to discuss differentialgeometric properties of $K^{n}$ corresponding to the Riemannian structure determined on it by a certain choice of a $d s^{2}$. We write the coordinates in $K^{n}$ as $z^{\mu}(1 \leqq \mu \leqq n)$; and we choose coordinates $\zeta^{i}(1 \leqq i \leqq r)$ on each one of the cells $K_{\lambda}^{r}(1 \leqq r \leqq n-1)$; for instance, we may choose the $\zeta^{i}$ from among the $z^{\mu}$, taking care to select such as are independent on $K_{\lambda}^{r}$, and this may be understood for definiteness, although playing no part in the sequel. In what follows, $N=n+q$ is any integer greater than or equal to $n$; and we make for $\$ \S 5-6$ the following conventions about the ranges of the various letters which will occur as indices:

$$
1 \leqq \alpha \leqq N ; 1 \leqq \mu, \nu \leqq n ; \quad 1 \leqq i, j \leqq r ; \quad 1 \leqq \rho \leqq q ; 1 \leqq \sigma \leqq n-r .
$$

We shall consider real-valued functions $\phi(z)$, defined on $K^{n}$. As usual, such a function is said to be of class $C^{1}$ (on $K^{n}$ ) if it has a differential. $d \phi=\sum_{\mu} \phi_{\mu}(z) \cdot d z^{\mu}$ with coefficients $\phi_{\mu}(z)=\partial \phi / \partial z^{\mu}$ which are continuous functions over $K^{n}$; class $C^{m}$ is defined inductively, $\phi$ being of class $C^{m}$ if it is of class $C^{1}$ and the $\partial \phi / \partial z^{\mu}$ are of class $C^{m-1}$.

Local properties of $K^{n}$ as a differentiable space are those which remain invariant under a differentiable change of local coordinates with jacobian different from 0 . Such properties include the intrinsic definition of the tangent affine space $T^{n}(z)$ and of the angle of $K^{n}$ at the point $z$ as follows. $T^{n}(z)$ is the vector-space consisting of all differentiations $X \phi$, defined over the set of all functions $\phi$ of class $C^{1}$ in a neighborhood of $z$, which can be expressed as $X \phi=\lim \xi \cdot\left[\phi\left(z^{\prime \prime}\right)-\phi\left(z^{\prime}\right)\right]$, where $z^{\prime}$ and $z^{\prime \prime}$ both tend to $z$ within $K^{n}$, and $\xi$ 
tends to $+\infty$. The vectors $X_{\mu} \phi=\partial \phi / \partial z^{\mu}$ form a basis for $T^{n}(z)$, so that every point of $T^{n}(z)$ can be written as $X \phi=\sum_{\mu} x^{\mu} \cdot \partial \phi / \partial z^{\mu}$; we shall denote by $x$ the point of $T^{n}(z)$ which, for that basis, has the components $x^{\mu}$. As in $\$ 2$, the dual space $\bar{T}^{n}(z)$ to $T^{n}(z)$ is the space of the linear forms $(y, x)=\sum_{\mu} y_{\mu} \cdot x^{\mu}$; the elements $x$ of $T^{n}(z)$ and $y$ of $\bar{T}^{n}(z)$ are known in tensor-calculus as contravariant and covariant vectors, respectively.

The angle of $K^{n}$ at $z$ is the subset of $T^{n}(z)$, consisting of all those differentiations $X \phi$ which can be expressed as $X \phi=\lim \xi \cdot\left[\phi\left(z^{\prime}\right)-\phi(z)\right]$, where $z^{\prime}$ tends to $z$ within $K^{n}$ and $\xi$ tends to $+\infty$; by the correspondence which maps every point $x=\left(x^{\mu}\right)$ in $T^{n}(z)$ onto the point with coordinates $x^{\mu}$ in the affine space $R^{n}$ containing $K^{n}$, that angle is transformed into the angle of $K^{n}$ at $z$ as defined in $\S 4$, the difference between the two being of course that the latter was defined in affine space whereas the definition of the former refers to $K^{n}$ as a differentiable space. The relationship between them implies that, if $z=z(\zeta)$ is an inner point of $K_{\lambda}^{r}$, having in $K_{\lambda}^{r}$ the coordinates $\zeta^{i}$, the angle at $z$ is of dimension $n$ and type $r$; we then denote it by $A_{r, \lambda}(\zeta)$; the linear manifold $T_{\lambda}^{r}(\zeta)$ contained in $A_{r, \lambda}(\zeta)$ will be identified as usual with the tangent affine space to $K_{\lambda}^{r}$ by the formulae $\partial \phi / \partial \zeta^{i}=\sum_{\mu} \partial \phi / \partial z^{\mu} \cdot \partial z^{\mu} / \partial \zeta^{i} ;$ it is spanned by the $r$ linearly independent vectors $\left(\partial z^{\mu} / \partial \zeta^{i}\right)$. We denote by $\bar{A}_{\lambda}^{n-r}(\zeta)$ the dual angle to $A_{r, \lambda}(\zeta)$, which is of dimension $n-r$ and type 0 ; it is contained in the linear manifold $N_{\lambda}^{n-r}(\zeta)$ of all vectors $y=\left(y_{\mu}\right)$ such that $(y, x)=0$ for $x$ in $T_{\lambda}^{r}(\zeta)$.

We now consider mappings $f(z)=\left(f^{\alpha}(z)\right)$ of $K^{n}$ into an affine space $R^{N} ; f(z)$ is said to be of class $C^{m}$ if each $f^{\alpha}(z)$ is of class $C^{m}$. A mapping $f(z)=\left(f^{\alpha}(z)\right)$ will be said to define an $n$-dimensional curved cell $\left(K^{n}, f\right)$ if it is of class $C^{1}$ and the $n$ vectors $\left(\partial f^{\alpha} / \partial z^{\mu}\right)$ in $R^{N}$ are linearly independent for every $z$ in $K^{n}$. As usual, the linear manifold spanned by the vectors $\left(\partial f^{\alpha} / \partial z^{\mu}\right)$ in $R^{N}$ is identified with the tangent affine space $T^{n}(z)$ to $K^{n}$ at $z$ by identifying point $x=\left(x^{\mu}\right)$ in $T^{n}(z)$ with the vector $\left(\sum_{\mu} x^{\mu} \cdot \partial f^{\alpha} / \partial z^{\mu}\right)$ in $R^{N} ; T^{n}(z)$ thus appears as imbedded in $R^{N}$. The manifold $T_{\lambda}^{r}(\zeta)$, as a submanifold of $T^{n}(z)$ when $z=z(\zeta)$ is in $K_{\lambda}^{r}$, is thus also imbedded in $R^{N}$, and as such is spanned by the vectors $\left(\partial f^{\alpha} / \partial \zeta^{i}\right)=\left(\sum_{\mu} \partial z^{\mu} / \partial \zeta^{i} \cdot \partial f^{\alpha} / \partial z^{\mu}\right)$. In the same imbedding, the angle $A_{r, \lambda}(\zeta)$ appears as an angle of dimension $n$ and type $r$ in $R^{N}$, contained in $T^{n}(z)$ and containing $T_{\lambda}^{r}(\zeta)$. As the vectors $\left(\partial f^{\alpha} / \partial \zeta^{i}\right)$ are independent, the mapping $f$, when restricted to $K_{\lambda}^{\top}$, defines a curved cell $\left(K_{\lambda}^{\top}, f\right)$ of dimension $r$ in $R^{N}$.

We now take $R^{N}$ as a Euclidean space; cartesian coordinates being chosen for convenience, the distance is defined by the form $(w, w)=\sum_{\alpha}\left(w^{\alpha}\right)^{2}$. The quadratic differential form $(d f, d f)=\sum_{\alpha}\left(d f^{\alpha}\right)^{2}=\sum_{\mu, \nu} g_{\mu \nu} \cdot d z^{\mu} d z^{\nu}$ is nondegenerate, under the assumptions made on $f$, and defines a Riemannian geometry on $K^{n}$; this amounts to making the tangent affine space $T^{n}(z)$ into a Euclidean space, either by means of its imbedding in $R^{N}$ or intrinsically by $(x, x)=\sum_{\mu, \nu} g_{\mu \nu} \cdot x^{\mu} x^{\nu}$; the $g_{\mu \nu}$ are functions of $z$ alone. We may then identify $T^{n}(z)$ with its dual $\bar{T}^{n}(z)$, as in $\S 3$, by the correspondence $y_{\mu}=\sum_{\nu} g_{\mu \nu} \cdot x^{\nu} ;$ calling, as usual, $\left\|g^{\mu \nu}\right\|$ the inverse matrix to $\left\|g_{\mu \nu}\right\|$, we have then $x^{\mu}=\sum_{\nu} g^{\mu \nu} \cdot y_{\nu}$; the 
$y_{\mu}$ are called the covariant components of the tangent vector $x$, and the quantities $\sum_{\mu} x^{\mu} \cdot \partial f^{\alpha} / \partial z^{\mu}$ are its components in $R^{N}$.

The Riemannian geometry thus defined in $K^{n}$ induces on each $K_{\lambda}^{\top}$ a Riemannian geometry, with the fundamental form $(d f, d f)=\sum_{i j} \gamma_{i j} \cdot d \zeta^{i} d \zeta^{i}$, where $\gamma_{i j}=\sum_{\mu \nu} g_{\mu \nu} \cdot \partial z^{\mu} / \partial \zeta^{i} \cdot \partial z^{\nu} / \partial \zeta^{i}$. The determinants of the matrices $\left\|g_{\mu \nu}\right\|,\left\|\gamma_{i j}\right\|$ are denoted by $g$ and $\gamma$, respectively; we have $g>0, \gamma>0$.

We now call $N^{q}(z)$ the orthogonal linear manifold to $T^{n}(z)$ in $R^{N}$, that is, the normal linear manifold to the cell at $z$; and, taking $z=z(\zeta)$ to be an inner point of $K_{\lambda}^{\top}$, we apply to $A_{r, \lambda}(\zeta)$ the results of $\S 3$. Identifying, as we now do, $\bar{T}^{n}(z)$ with $T^{n}(z)$, the dual linear manifold $N_{\lambda}^{n-r}(\zeta)$ to $T_{\lambda}^{r}(\zeta)$ within $T^{n}(z)$ appears as the orthogonal manifold to $T_{\lambda}^{\tau}(\zeta)$ within $T^{n}(z)$, that is, the normal manifold to the subcell $\left(K_{\lambda}^{r}, f\right)$; the orthogonal manifold to $T_{\lambda}^{r}(\zeta)$ within $R^{N}$ is then $N_{\lambda}^{n-r}(\zeta)+N^{q}(z)$. The dual angle $\bar{A}_{\lambda}^{n-r}(\zeta)$ to $A_{r, \lambda}(\zeta)$ within $T^{n}(z)$ is now an angle of dimension $n-r$ and type 0 in the normal manifold $N_{\lambda}^{n-r}(\zeta)$; it is the same as the dual, taken within $N_{\lambda}^{n-r}(\zeta)$, of the trace of $A_{r, \lambda}(\zeta)$ on $N_{\lambda}^{n-r}(\zeta)$. Finally, the dual $\bar{A}_{\lambda}^{N-r}(\zeta)$ of $A_{r, \lambda}(\zeta)$ within $R^{N}$ is an angle of dimension $N-r$ and type $q$, and can be written as $\bar{A}_{\lambda}^{N-r}(\zeta)=\bar{A}_{\lambda}^{n-r}(\zeta)+N^{q}(z)$; this means that a vector $w$ is in $\bar{A}_{\lambda}^{N-r}(\zeta)$ if, and only if, its orthogonal projection on $T^{n}(z)$ is in $\bar{A}_{\lambda}^{n-r}(\zeta)$.

It should be observed that the dual angle $\bar{A}_{\lambda}^{n-r}(\zeta)$ to $A_{r, \lambda}(\zeta)$, as originally defined in the dual affine space $\bar{T}^{n}(z)$ to $T^{n}(z)$, depends only upon $K^{n}$ regarded as a differentiable space, irrespective of the choice of $f$ or of a Riemannian structure; and we write that a vector $y$ in $\bar{T}^{n}(z)$, given by its components $y_{\mu}$, is in $\bar{A}_{\lambda}^{n-r}(\zeta)$ by writing that $\sum_{\mu} y_{\mu} \cdot X\left(z^{\mu}\right) \leqq 0$ for every differentiation $X$ contained in the angle of $K^{n}$ at $z(\zeta)$. On the other hand, the angles in $R^{N}$ and in $T^{n}(z)$ which we have identified with $\bar{A}_{\lambda}^{n-r}(\zeta)$, and which, for short, we also denote by the same symbol, depend, the former upon the choice of the mapping $f$, the latter merely upon the $g_{\mu \nu}$.

We now define the tube $\tau\left(K^{n}, f\right)$ of the curved cell $\left(K^{n}, f\right)$ as the subset of $K^{n} \times B^{N}$ which consists of all points $(z, w)$ of that product such that, if $z$ is an inner point of $K_{\lambda}^{\top}$ and $z=z(\zeta)$, then $w$ is in $\bar{A}_{\lambda}^{N-r}(\zeta)$. Whenever $f$ is an affine mapping, that is, when the $f^{\alpha}$ are linear functions, the tube $\tau\left(K^{n}, f\right)$ is the same as the tube $T\left(L^{n}\right)$ of the convex cell $L^{n}=f\left(K^{n}\right)$, as defined in $\S 4$. Furthermore, if $\left(K^{n}, f\right)$ is an arbitrary cell, the set $\Theta_{\delta}\left(K^{n}, f\right)$ of all points at a set-theoretical distance $\delta$ from $f\left(K^{n}\right)$ in $R^{N}$ is easily shown to be the same as the set of all points $y^{\alpha}=f^{\alpha}(z)+\delta \cdot w^{\alpha}$ when $(z, w)$ describes $\tau\left(K^{n}, f\right)$, and it seems very likely that these relations define a one-to-one correspondence between $\Theta_{\delta}\left(K^{n}, f\right)$ and $T\left(K^{n}, f\right)$ provided $f$ itself is a one-to-one mapping and provided $\delta$ is sufficiently small.

The central result of this paper is now implicit in the following lemma, which will turn out to contain the Gauss-Bonnet formula for curved cells: 
Lemma 5. The mapping $(z, w) \rightarrow w$ of the tube $T\left(K^{n}, f\right)$ into $B^{N}$ has everywhere the degree 1.

The lemma has been proved in $\$ 4$ for the Euclidean tube of a convex cell. The general case will be reduced to that special case by continuous deformation.

As a preliminary step, we consider the topological space, each point of which consists of a point $z$ in $K^{n}$ and a set of $q$ mutually orthogonal unitvectors in $N^{q}(z)$. This is a fibre-space over $K^{n}$, the fibre being homeomorphic to the group of all orthogonal matrices of order $q$; therefore, by Feldbau's theorem $\left.{ }^{9}\right)$, it is the direct product of $K^{n}$ with the fibre; that implies that it is possible to choose the $q$ vectors $n_{\rho}(z)$ as continuous functions of $z$ in $K^{n}$ so as to satisfy the above conditions for every $z$. We call $n_{\rho}^{\alpha}(z)$ the components of $n_{\rho}(z)$ in $R^{N}$.

Let now $z=z(\zeta)$ be an inner point of $K_{\lambda}^{\top}$, and $w$ a point in $R^{N}$; call $x, u$ the orthogonal projections of $w$ on $T^{n}(z)$ and $N^{q}(z)$, respectively; call $x_{\mu}$ the covariant components of $x, u^{\rho}$ the components of $u$ with respect to the basisvectors $n_{p}(z)$, so that we have

$$
w^{\alpha}=\sum_{\mu, \nu} \frac{\partial f^{\alpha}}{\partial z^{\mu}} \cdot g^{\mu \nu} \cdot x_{\nu}+\sum_{\rho} n_{\rho}^{\alpha}(z) \cdot u^{\rho} .
$$

We have, then, $(w, w)=(x, x)+(u, u)=\sum_{\mu, \nu} g^{\mu \nu} \cdot x_{\mu} x_{\nu}+\sum_{\rho}\left(u^{\rho}\right)^{2} ;$ and $(z, w)$ is in the tube $T\left(K^{n}, f\right)$ if and only if $x$ is in $\bar{A}_{\lambda}^{n-r}(\zeta)$ and $(w, w) \leqq 1$.

All that applies to the special case when $f^{\alpha}(z)$ is replaced by $\sum_{\mu} \delta_{\mu}^{\alpha} \cdot z^{\mu}$, that is, by $z^{\mu}$ for $\alpha=\mu \leqq n$ and by 0 for $\alpha>n$, in which case the tube becomes the Euclidean tube $\Theta^{N}$ of a convex cell; therefore, $z=z(\zeta)$ being again an inner point of $K_{\lambda}^{T},(z, v)$ will be in $\Theta^{N}$ if and only if the vector in $\bar{T}^{n}(z)$ with the components $v_{\mu}=v^{\mu}(1 \leqq \mu \leqq n)$ is in $\bar{A}_{\lambda}^{n-r}(\zeta)$, and $\sum_{\alpha}\left(v^{\alpha}\right)^{2} \leqq 1$. Writing, therefore,

$$
\begin{aligned}
x_{\mu}=\left(\frac{\sum_{\mu, \nu} g^{\mu \nu}(z) \cdot v_{\mu} v_{\nu}}{\sum_{\mu} v_{\mu}^{2}}\right)^{-1 / 2} \cdot v_{\mu} & (1 \leqq \mu \leqq n), \\
u^{\rho}=v^{n+\rho} & (1 \leqq \rho \leqq q)
\end{aligned}
$$

these formulae, together with the formulae above, define a homeomorphic correspondence between the points $(z, v)$ of the Euclidean tube $\Theta^{N}$ and the points $(z, w)$ of $\tau\left(K^{n}, f\right)$.

We now assume coordinates to be such that 0 is in $K^{n}$; calling $\tau$ a parameter taking the values $0 \leqq \tau \leqq 1$, the point $\tau \cdot z=\left(\tau \cdot z^{\mu}\right)$ is in $K^{n}$ if $z$ is in $K^{n}$. For every $\tau>0$, we consider the curved cell $\left(K^{n}, \bar{f}\right)$ defined by $\bar{f}(z)=f(\tau \cdot z) / \tau$. Putting $\partial f^{\alpha} / \partial z^{\mu}=f_{\mu}^{\alpha}(z)$, we have, for the cell $\left(K^{n}, \bar{f}\right), \partial \bar{f}^{\alpha} / \partial z^{\mu}=f_{\mu}^{\alpha}(\tau \cdot z)$, $\bar{g}_{\mu \nu}(z)=g_{\mu \nu}(\tau \cdot z), \bar{g}^{\mu \nu}(z)=g^{\mu \nu}(\tau \cdot z)$, and we may take as normal vectors to that

( $)$ J. Feldbau, Sur la classification des espaces fibres, C. R. Acad. Sci. Paris vol. 208 (1939) p. 1621. 
cell $\bar{n}_{\rho}(z)=n_{\rho}(\tau \cdot z)$. That being so, the above formulae for the transformation of $\Theta^{N}$ into $\tau\left(K^{n}, \bar{f}\right)$ show that this transformation depends continuously upon $\tau$, and therefore that the tube $\tau\left(K^{n}, \bar{f}\right)$ is deformed continuously when $\tau$ varies. When $\tau$ tends to 0 , these formulae tend to the corresponding formulae for the cell $\left(K^{n}, f_{0}\right)$ defined by $f_{0}^{\alpha}(z)=\sum_{\mu} f_{\mu}^{\alpha}(0) \cdot z^{\mu}$ when the normal vectors for $\left(K^{n}, f_{0}\right)$ are taken as $n_{\rho}^{0}(z)=n_{\rho}(0) ; f_{0}$ being affine, $\left(K^{n}, f_{0}\right)$ is a convex cell. to which the results of $\$ 4$ apply.

Lemma 5 follows easily. For the image of our tube in $B^{N}$ by the mapping $(z, w) \rightarrow w$ is deformed continuously when the tube is so deformed; the image of its boundary remains in $S^{N-1}$. The degree is therefore constant during the deformation; as it is +1 for $\tau=0$, it is +1 for $\tau=1$, which was to be proved.

6. The Gauss-Bonnet formula for imbedded cells. We put

$$
d w=d w^{1} \cdot d w^{2} \cdots d w^{N} .
$$

A special consequence of Lemma 5 in $\S 5$ is that the integral of $d w$ over the tube $\tau\left(K^{n}, f\right)$ is equal to the integral of the same differential form over $B^{N}$, that is, to the volume $v\left(B^{N}\right)$ of the interior of the unit-sphere in $R^{N}$. Therefore, calling $I_{r, \lambda}$ the integral of $d w / v\left(B^{N}\right)$ over the set of those points $(z, w)$ in the tube for which $z$ is an inner point of $K_{\lambda}^{r}$, we have

$$
\sum_{r=0}^{n} \sum_{\lambda} I_{r, \lambda}=1
$$

This becomes the Gauss-Bonnet formula when the $I_{r, \lambda}$ are expressed intrinsically in terms of the Riemannian geometry on $K^{n}$. The calculation depends upon a lemma which immediately follows from a formula proved in a recent paper by $\mathrm{H}$. Weyl $\left({ }^{10}\right)$.

LEMMA 6. Let $\left\|\Lambda_{i j}\right\|,\left\|L_{i j}^{p}\right\|$ be $q+1$ matrices of order $r$; and write

$$
\mathbf{P}_{i_{1} i_{2} j_{1} j_{2}}=\sum_{\rho}\left(L_{i_{1} j_{1}}^{\rho} \cdot L_{i_{2} j_{2}}^{\rho}-L_{i_{1} j_{2}}^{\rho} \cdot L_{i_{2} j_{1}}^{\rho}\right) \text {. }
$$

Then the integral of $\left|\Lambda_{i j}+\sum_{\rho} L_{i j}^{\rho} \cdot u^{\rho}\right| \cdot d u^{1} \cdot d u^{2} \cdots d u^{q}$, taken over the volume $\sum_{\rho}\left(u^{\rho}\right)^{2} \leqq c^{2}$, is equal to:

$$
\begin{aligned}
v\left(B^{q}\right) & \cdot \sum_{f=0}^{[r / 2]} k_{q, f} \cdot c^{q+2 f} \\
& \cdot \sum_{i, j} \frac{\epsilon^{(i)} \epsilon^{(j)}}{2^{2 f} \cdot f !(r-2 f) !} \mathbf{P}_{i_{1} i_{2} j_{1} j_{2}} \cdots \mathbf{P}_{i_{2 f-1} i_{2} j_{2 f-1} j_{2 f}} \Lambda_{i_{2 f+1} i_{2 f+1}} \cdots \Lambda_{i_{r} i_{r}}
\end{aligned}
$$

where $k_{q, f}=1 /(q+2)(q+4) \cdots(q+2 f)$, and the conventions about summation are as explained in $\$ 1$.

(10) Loc. cit., Footnote 7, p. 470. Similar calculations may also be found in W. Killing. Die nicht-euklidischen Raumformen in analytischer Behandlung, Teubner, Leipzig, 1885, p. 255. 
Our calculation of $I_{r, \lambda}$ will be valid under the assumption that the mapping $f(z)$ is of class $C^{2}$; in order, however, to be able to introduce the Riemannian curvature tensor, we assume from now onwards that $f(z)$ is of class $C^{3}$. In the course of the calculation of $I_{r, \lambda}$, we simplify notations by omitting the subscript $\lambda$.

We may calculate $I_{r}$ by cutting up the cell $K^{r}$ into small subsets, and cutting up $I_{r}$ correspondingly; we take those subsets to be cells of a subdivision of $K^{r}$, and so small that it is possible to define, on each of them, $q$ vectors $n_{\rho}(\zeta)$ of class $C^{1}$ and $n-r$ vectors $\nu_{\sigma}(\zeta)$, also of class $C^{1}$, satisfying the following conditions: the $n_{\rho}(\zeta)$ are an orthonormal basis for the normal linear manifold $N^{q}(z)$ at $z(\zeta)$; the $\nu_{\sigma}(\zeta)$ are an orthonormal basis for the normal manifold $N^{n-r}(\zeta)$ to $T^{r}(\zeta)$ in $T^{n}(z)$; and, calling $\nu_{\sigma}^{\alpha}$, $n_{\rho}^{\alpha}$ the components of those vectors in $R^{N}$, the matrix $\Delta=\left\|\partial f^{\alpha} / \partial \zeta^{i} \nu_{\sigma}^{\alpha} n_{\rho}^{\alpha}\right\|$ has a determinant greater than 0 . The latter determinant can then be calculated by observing that, if $\Delta^{T}$ is the transpose of $\Delta$ and $\Gamma=\left\|\gamma_{i j}\right\|$, we have

$$
\Delta^{T} \cdot \Delta=\left\|\begin{array}{ll}
\Gamma & 0 \\
0 & 1
\end{array}\right\|
$$

and therefore $(|\Delta|)^{2}=\gamma$, so that $|\Delta|=+\gamma^{1 / 2}$.

$z=z(\zeta)$ belonging to one of our subsets in $K^{r}$, let $(z, w)$ be in the tube $\tau\left(K^{n}, f\right)$. Let $x, u$ be the orthogonal projections of $w$ on $T^{n}(z)$ and $N^{q}(z)$, respectively; $x$ is in $\bar{A}^{n-r}(\zeta) \subset N^{n-r}(\zeta)$, so that $x$ can be written as $\sum_{\sigma} \nu_{\sigma}(\zeta) \cdot t^{\sigma}$; let $u^{\rho}$ be the components of $u$ with respect to the basis $n_{\rho}$. We have:

$$
w^{\alpha}=\sum_{\sigma} v_{\sigma}^{\alpha}(\zeta) \cdot t^{\sigma}+\sum_{\rho} n_{\rho}^{\alpha}(\zeta) \cdot u^{\rho} .
$$

As these are functions of $\zeta, t, u$ of class $C^{1}$, we can express $d w$ in terms of $d \zeta=d \zeta^{1} \cdot d \zeta^{2} \cdots d \zeta^{r}, d t=d t^{1} \cdot d t^{2} \cdots d t^{n-r}, d u=d u^{1} \cdot d u^{2} \cdots d u^{q}$ :

$$
d w=\left|\sum_{\sigma} \frac{\partial \nu_{\sigma}^{\alpha}}{\partial \zeta^{i}} \cdot t^{\sigma}+\sum_{\rho} \frac{\partial n_{\rho}^{\alpha}}{\partial \zeta^{i}} \cdot u^{\rho} \nu_{\sigma}^{\alpha} n_{\rho}^{\alpha}\right| \cdot d \zeta \cdot d t \cdot d u \text {. }
$$

The determinant is best calculated by multiplying its matrix to the left by $\Delta^{T}$, the determinant of which has been found to be $+\gamma^{1 / 2}$; that gives a matrix of the form

$$
\left\|\begin{array}{cc}
M & 0 \\
* & 1
\end{array}\right\|
$$

which has the determinant $|M|$. That gives:

if we put

$$
d w=\left|\Lambda_{i j}+\sum_{\rho} L_{i j}^{\rho} \cdot u^{\rho}\right| \cdot \gamma^{-1 / 2} d \zeta \cdot d t \cdot d u
$$




$$
\Lambda_{i j}=\sum_{\alpha, \sigma} \frac{\partial f^{\alpha}}{\partial \zeta^{i}} \cdot \frac{\partial \nu_{\sigma}^{\alpha}}{\partial \zeta^{j}} \cdot t^{\sigma}, \quad L_{i j}^{\rho}=\sum_{\alpha} \frac{\partial f^{\alpha}}{\partial \zeta^{i}} \cdot \frac{\partial n_{\rho}^{\alpha}}{\partial \zeta^{j}} .
$$

In the integration of this, orientation has to be considered. Call $t, u$ the points with the coordinates $\left(t^{\sigma}\right),\left(u^{p}\right)$, respectively, in two auxiliary spaces $P^{n-r}, P^{q}$; we also consider the point with the coordinates $\zeta^{i}, t^{\sigma}, u^{\rho}$ in the space $P^{N}=K^{r} \times P^{n-r} \times P^{q}$. The formulae $z=z(\zeta), w=\sum_{\sigma} \nu_{\sigma} \cdot t^{\sigma}+\sum_{\rho} n_{\rho} \cdot u^{\rho}$ define the portion of the tube now under consideration as a homeomorphic image of class $C^{1}$ of the subset of $P^{N}$ defined as follows: $\zeta$ is in a given subset of $K^{r} ; t$ is such that $x=\sum_{\sigma} \nu_{\sigma} \cdot t^{\sigma}$ is in $\bar{A}^{n-r}(\zeta)$; and $\sum_{\sigma}\left(t^{\sigma}\right)^{2}+\sum_{\rho}\left(u^{\rho}\right)^{2} \leqq 1$. As $\bar{A}^{n-r}$ depends continuously upon $\zeta$, that set is the closure of an open set in $P^{N}$. Call now $o_{1}, o_{2}, o_{3}$ any orientations of $K^{r}, P^{n-r}, P^{q}$, respectively; the factors in the product $P^{N}=K^{r} \times P^{n-r} \times P^{q}$ being ordered as written, $o_{1}, o_{2}, o_{3}$ define an orientation $o_{1} \times O_{2} \times O_{3}$ in $P^{N}$, and therefore a local orientation, also denoted by $o_{1} \times o_{2} \times o_{3}$, in the part of the tube which we are discussing. On the other hand, the mappings $(t) \rightarrow \sum_{\sigma \nu_{\sigma}} \cdot t^{\sigma},(u) \rightarrow \sum_{\rho} n_{\rho} \cdot u^{\rho}$ of $P^{n-r}, P^{q}$ onto $N^{n-r}(\zeta), N^{q}(z)$ transform $o_{2}, o_{3}$ into orientations, also denoted by $o_{2}, o_{3}$, of $N^{n-r}(\zeta), N^{q}(z)$. We now choose for $o_{1}, o_{2}, o_{3}$ the natural orientations of $K^{r}, P^{n-r}, P^{q}$, respectively, defined by the coordinates $\zeta^{i}, t^{\sigma}, u^{\rho}$ taken in each case in their natural order. The condition on the sign of $|\Delta|$ which served to define the $\nu_{\sigma}, n_{\rho}$ amounts to saying that the orientations $o_{1}, o_{2}, o_{3}$ of $f\left(K^{r}\right), N^{n-r}(\zeta), N^{q}(z)$ at $z=z(\zeta)$ define, when taken in that order, the natural orientation of $R^{N}$. That being so, we now show that the local orientation of the tube defined as $o_{1} \times o_{2} \times o_{3}$ coincides with that orientation $\Omega$ of the tube as a whole which ensures the validity of Lemma 5 . That is easily verified for the tube of a convex cell, by identifying it with a subset $\Theta^{N}$ of $R^{N}$ as in $\S 4$. In the general case we use the deformation of our tube into that of a convex cell, by means of which we proved Lemma 5 ; for, in such a deformation, the manifolds $N^{n-r}(\zeta), N^{q}(z)$ vary continuously, and therefore we have $o_{1} \times o_{2} \times o_{3}=\Omega$ during the whole deformation, since this is true for one value $\tau=0$ of the parameter.

We can now proceed to integrate $d w$ by first integrating with respect to $u$ while $\zeta$ and $t$ are kept constant; $u$ is to be given all values such that $\sum_{\rho}\left(u^{\rho}\right)^{2} \leqq 1-\sum_{\sigma}\left(t^{\sigma}\right)^{2}$. We first observe that, by differentiating the relations $\sum_{\alpha} \partial f^{\alpha} / \partial \zeta^{i} \cdot \nu_{\sigma}^{\alpha}=0, \sum_{\alpha} \partial f^{\alpha} / \partial \zeta^{i} \cdot n_{\rho}^{\alpha}=0$ which express that $\nu_{\sigma}, n_{\rho}$ are normal vectors to $T^{r}(\zeta)$, we get the following expressions for $\Lambda_{i j}, L_{i j}^{\rho}$ :

$$
\Lambda_{i j}=-\sum_{\alpha} \frac{\partial^{2} f^{\alpha}}{\partial \zeta^{i} \partial \zeta^{j}} \cdot x^{\alpha}, \quad L_{i j}^{\rho}=-\sum_{\alpha} \frac{\partial^{2} f^{\alpha}}{\partial \zeta^{i} \partial \zeta^{j}} \cdot n_{\rho}^{\alpha}
$$

where $x^{\alpha}=\sum_{\sigma} \nu_{\sigma}^{\alpha} \cdot t^{\sigma}$ are the components of the vector $x$ in $R^{N}$; these are the negatives of coefficients of the so-called "second fundamental forms" of $f\left(K^{r}\right)$ in $R^{N}$. The $\Lambda_{i j}$ are thus seen not to depend upon the choice of the basis-vectors $\nu_{\sigma}$ in $N^{n-r}(\zeta)$, but only upon the vector $x$; as such, we shall now call them $\Lambda_{i j}(x)$; it is known that they are intrinsic quantities with respect to the 
Riemannian geometry in $K^{n}$, and can be expressed by formula (3) in $\$ 1$, if we denote by $x_{\mu}$ the covariant components of $x$; we have $x_{\mu}=\sum_{\alpha} \partial f^{\alpha} / \partial z^{\mu} \cdot x^{\alpha}$.

The application of Lemma 6 further leads to the introduction of the quantities

$$
\mathbf{P}_{i_{1} i_{2} j_{1} j_{2}}=\sum_{\rho}\left(L_{i_{1} j_{1}}^{\rho} \cdot L_{i_{2} j_{2}}^{\rho}-L_{i_{1} j_{2}}^{\rho} \cdot L_{i_{2} j_{1}}^{\rho}\right),
$$

which also are known to be intrinsic quantities, their expression in terms of the curvature tensor in $K^{n}$ being given by formula (2) in $\$ 1$. We now distinguish two cases:

(a) If $r=n$, the $\zeta^{i}$ in the foregoing calculation should be read as $z^{\mu}$, and $\gamma$ as $g$; there are no $\nu_{\sigma}$, no $t^{\sigma}$, no $\Lambda_{i j}$. Integrating $d w$ first with respect to $u$, we get, by straightforward application of Lemma 6 :

$$
I_{n}=\int_{K^{n}} \Psi(z) d v(z),
$$

where $\Psi(z)$ is defined by formula (1) in $\$ 1$.

(b) If $r<n$, the integration of $d w$ with respect to $u$ by Lemma 6 gives, if we define the functions $\Phi_{r, f}(\zeta, x)$ by formula (4) in $\$ 1$

$$
v\left(B^{q}\right) \cdot \sum_{f=0}^{[r / 2]} k_{q, f} \cdot\left[1-\sum_{\sigma}\left(t^{\sigma}\right)^{2}\right]^{(q / 2)+f} \cdot \Phi_{r, f}(\zeta, x) \cdot d v(\zeta) \cdot d t,
$$

where $d v(\zeta)=\gamma^{1 / 2} \cdot d \zeta$ is the intrinsic volume-element in $K^{r}$. We may push the integration one step further, by writing $x=a \cdot \xi, x_{\mu}=a \cdot \xi_{\mu}, t^{\sigma}=a \cdot \tau^{\sigma}$, where $\sum_{\sigma}\left(\tau^{\sigma}\right)^{2}=1$ and $0 \leqq a \leqq 1 ; \xi_{\mu}$ are thus the covariant components of vector $\xi$, $\tau^{\sigma}$ its components with respect to the basis $\nu_{\sigma}$, and $\xi$ is on the unit-sphere in $N^{n-r}(\zeta) ; \xi$ describes a spherical cell $\Gamma(\zeta)$, the trace on that sphere of $\bar{A}^{n-r}(\zeta)$; $\Gamma(\zeta)$ is the outer angle in $N^{n-r}(\zeta)$ of the trace of $A_{r}(\zeta)$ on $N^{n-r}(\zeta)$. Calling $d \xi$ the area-element or spherical measure on that sphere, we have $d t=a^{r-1} \cdot d a \cdot d \xi$. We can now carry out the integration in $a$, which involves only the elementary integral $\int_{0}^{1}\left(1-a^{2}\right)^{(q / 2)+f} \cdot a^{n-2 f-1} \cdot d a$, and thus find

$$
I_{r}=\int_{K^{r}} d v(\zeta) \int_{\Gamma(\zeta)} \Psi\left(\zeta, \xi \mid K^{r}\right),
$$

where $\Psi$ is defined by formula (5) in $\$ 1$. This, combined with our earlier result $\sum_{r, \lambda} I_{r, \lambda}=1$, completes the proof of Theorem II for $K^{n}$, with the Riemannian structure defined by the $g_{\mu \nu}$, if we observe that the inner characteristic of $K^{n}$ is $\chi^{\prime}\left(K^{n}\right)=(-1)^{n}$.

It may be observed that, for $r=n-1$, the outer angle $\Gamma(\zeta)$ is reduced to a point, namely, the unit-vector $\xi$ on the outer normal to $K^{n-1}$ in the tangent space to $K^{n}$; the integral in $d \xi$ should then be understood to mean the value of the integrand at that point. Similarly, for $r=0, K^{r}$ is reduced to a point, 
and the integral in $d v(\zeta)$ should be understood correspondingly. In the latter case, $I_{0}$ contains only one term, corresponding to $f=0$, which is simply the spherical measure of the outer angle $\Gamma(\zeta)$, measured with the area of the sphere taken as the unit. In the case of a Euclidean convex cell, the terms $I_{0}$ in our formula are the only ones which do not reduce to 0 .

As a preparation to $\$ 7$, we furthermore have to prove some identities concerning the application of the above results to cells of lower dimension imbedded in $K^{n}$. Let $L^{p}$ be a convex cell, $\phi$ a one-to-one mapping of class $C^{3}$ of $L^{p}$ into $K^{n}$, such that $\left(L^{p}, \phi\right)$ is a curved cell; we assume that $0 \leqq p \leqq n-1$. For simplicity of notations, we identify $L^{p}$ with its image in $K^{n}$ by $\phi$, and call $\left(L^{p}, f\right)$ the curved cell which according to earlier conventions should be written as $\left(L^{p}, h\right)$ where $h$ is the product of the two mappings $f, \phi . L^{r}$ denoting either $L^{p}$ or, for $0 \leqq r \leqq p-1$, any one of the boundary cells of $L^{p}$, we choose coordinates $\zeta^{i}$ on $L^{r}$, and again identify $L^{r}$ with its image in $K^{n}$. The part of the tube of $\left(L^{p}, f\right)$ which corresponds to $L^{r}$ then consists of all points $(z, w)$ in $K^{n} \times B^{N}$ for which $z=z(\zeta)$ is an inner point of $L^{r},(w, w) \leqq 1$, and $w$ is in the dual in $R^{N}$ to the angle of $L^{p}$ at this point; the latter angle is in the tangent linear manifold to $L^{p}$, which as before should be considered as imbedded in the tangent linear manifold $T^{n}(z)$ to $K^{n}$ at the same point, and is of dimension $p$ and type $r$; we denote it by $B_{r}(\zeta)$. Let $N^{n-r}(\zeta)$ be the normal linear manifold to $L^{r}$ at $\zeta$; the dual to $B_{r}(\zeta)$ in $R^{N}$ consists of all vectors $w$ whose projection $x$ on $T^{n}(z)$ belongs to the dual to $B_{r}(\zeta)$ in $T^{n}(z)$, which is contained in $N^{n-r}(\zeta)$. Let $L^{\prime r}$ be an open subset of $L^{r}$, so small that we may define on it $q$ vectors $n_{\rho}$ and $n-r$ vectors $\nu_{\sigma}$ precisely as before ( $K^{r}$ being replaced by $L^{r}$ ). The calculation and integration of $d w$ for that part of the tube consisting of all points $(z, w)$ with $z$ in $L^{\prime r}$ now proceeds, without any change, just as before; the case $r=n$ does not arise, as $r \leqq p \leqq n-1$; calling $I_{r}^{\prime}$ the integral of $d w / v\left(B^{N}\right)$ over that part of the tube, we have, therefore:

$$
I_{r}^{\prime}=\int_{L^{\prime r}} d v(\zeta) \int_{\Gamma\left(L^{p}, \zeta\right)} \Psi\left(\zeta, \xi \mid L^{r}\right)
$$

where we now denote by $\Gamma\left(L^{p}, \zeta\right)$ the trace on the unit-sphere in $N^{n-r}(\zeta)$ of the dual to $B_{r}(\zeta)$ in $T^{n}(z)$. On the other hand, we could have applied our method to $L^{p}$ itself, considered intrinsically and not as imbedded in $K^{n}$; this, for $r=p$, would have given us

$$
I_{p}^{\prime}=\int_{L^{\prime p}} \Psi_{0}(\zeta) d v(\zeta)
$$

if we denote by $\Psi_{0}(\zeta)$ the invariant built up in $L^{p}$ just as $\Psi(z)$ was built up in $K^{n}$. As this is true for any sufficiently small $L^{\prime p}$, we get, for every inner point $\zeta$ of $L^{p}$, the identity 


$$
\Psi_{0}(\zeta)=\int_{\Gamma\left(L^{p}, \zeta\right)} \Psi\left(\zeta, \xi \mid L^{p}\right)
$$

where $\Gamma\left(L^{p}, \zeta\right)$ being as above defined, is easily seen to be the full sphere in $N^{n-p}(\zeta)$. Similarly, for $0 \leqq r \leqq p-1$, we denote by $\Psi_{0}\left(\zeta, \xi_{0} \mid L^{r}\right)$ the quantity, similar to $\Psi$, which is built up in $L^{p}$ from the Riemannian structure defined on $L^{p}$ by its imbedding in $K^{n}$, from the imbedded submanifold $L^{r}$, and from a unit-vector $\xi_{0}$ normal to $L^{r}$ in the tangent linear manifold $T^{p}(\zeta)$ to $L^{p}$; and calling $\Gamma_{0}\left(L^{p}, \zeta\right)$ the trace on the unit-sphere of the dual to $B_{r}(\zeta)$ in $T^{p}(\zeta)$, we get as before, $\zeta$ being any inner point of $L^{r}$ :

$$
\int_{\Gamma_{0}\left(L^{p}, \zeta\right)} \Psi_{0}\left(\zeta, \xi_{0} \mid L^{r}\right)=\int_{\Gamma\left(L^{p}, \zeta\right)} \Psi\left(\zeta, \xi \mid L^{r}\right)
$$

The identities (6), (7) contain only quantities which are intrinsic in $K^{n}$ for the Riemannian structure defined in $K^{n}$ by the metric tensor $g_{\mu \nu}$. They have just been proved for the case in which the $g_{\mu \nu}$ are defined by a mapping $f$ of $K^{n}$ into $R^{N}$; however, they depend only upon the $g_{\mu \nu}$ and their derivatives of the first and second order at point $z(\zeta)$. It is easy to define a small cell $K^{\prime n}$ containing a neighborhood of point $z(\zeta)$ in $K^{n}$, and a mapping $f^{\prime}$ of $K^{\prime n}$ into a Euclidean $R^{N^{\prime}}$, so that $\left(K^{\prime n}, f^{\prime}\right)$ is a curved cell and that the $g_{\mu \nu}^{\prime}$ defined by $f^{\prime}$ over $K^{\prime n}$ have, together with their derivatives of first and second order, prescribed values at $z(\zeta)$; in fact, we may do that by taking any analytic $g_{\mu \nu}^{\prime}$ satisfying the latter conditions, and apply Cartan's theorem $\left({ }^{4}\right)$, but there are of course more elementary methods of obtaining the same result. As (6), (7) are purely local properties of the Riemannian cell $K^{n}$ and of the imbedded $L^{p}, L^{r}$, they are thus shown to hold without any restriction. They could, of course, be verified by direct calculation; this would be straightforward but cumbersome, and would require another application of Lemma 6.

7. The Gauss-Bonnet formula for Riemannian polyhedra. We first define Riemannian polyhedra as follows.

Let $P^{n}$ be a compact connected topological space, for which there has been given a covering by open subsets $\Omega_{\imath}$ and a homeomorphic mapping $\phi_{\text {، }}$ of each $\Omega_{\imath}$ onto an $n$-dimensional convex angle $C_{\imath}$ which may be $R^{n}$; if the $\phi_{\imath}$ and the inverse mappings $\psi_{\imath}$ are such that every $\phi_{k}\left[\psi_{\imath}(x)\right]$ is of class $C^{m}$ at every $x \in C_{\text {، }}$ such that $\psi_{\iota}(x) \in \Omega_{\kappa}, P^{n}$ will be called an $n$-dimensional differentiable polyhedron of class $C^{m}$. As noted before $(\$ 2)$, re-entrant angles would lend themselves to similar treatment but are purposely avoided for simplicity's sake.

By a differentiable cell of class $C^{m}$, we understand a differentiable polyhedron of class $C^{m}$ which can be put into a one-to-one correspondence of class $C^{m}$ with a convex cell.

The beginning of $\$ 5$ provides a definition for the tangent affine space and the angle of a differentiable cell at any one of its points; those definitions, 
being purely local, apply without any change to a differentiable polyhedron. If $C$ is the angle of $P^{n}$ at the point $z, z$ has a neighborhood homeomorphic to $C$; if $C$ is of type $r$, we say that $z$ is of type $r$ in $P^{n}$. Points of type $n$ in $P^{n}$ are called inner points of $P^{n}$. Points of type at most $r$ (where $0 \leqq r \leqq n$ ) form a closed, and therefore compact, subset of $P^{n}$, the closure of the set of the points of type $r$; if the latter consists of $N_{r}$ connected components, the former is the union of $N_{r}$, and not of less than $N_{r}$, differentiable polyhedra $P_{\lambda}^{r}$ of dimension $r$. A point of type $r$ is an inner point of one of the $P_{\lambda}^{r}$, and of no other $P_{\mu}^{s}$; if an inner point of $P_{\lambda}^{r}$ is contained in $P_{\mu}^{s}$, then $P_{\lambda}^{r} \subset P_{\mu}^{s}$. The $P_{\lambda}^{r}$, for $0 \leqq r \leqq n-1$, will be called the boundary polyhedra of $P^{n}$.

By a regular subpolyhedron $Q^{p}$ in $P^{n}$, we understand the one-to-one image of a polyhedron $Q_{0}^{p}$ in $P^{n}$, provided it satisfies the following conditions: $\zeta^{i}$ being local coordinates in $Q_{0}^{p}$ at any point, and $z^{\mu}$ local coordinates in $P^{n}$ at the image of that point, the functions $z^{\mu}(\zeta)$ which locally define the mapping are of the same class $C^{m}$ as the polyhedron $P^{n}$, and the matrix $\left\|\partial z^{\mu} / \partial \zeta^{i}\right\|$ is of rank $p$. Each boundary polyhedron $P_{\lambda}^{r}$ of $P^{n}$ is a regular subpolyhedron of $P^{n}$.

We say that a finite set of distinct regular subpolyhedra $Q_{p}^{r}$ of $P^{n}$ forms a subdivision $\mathscr{D}$ of $P^{n}$ if the following conditions are fulfilled: (a) each point of $P^{n}$ is an inner point of at least one $Q_{\rho}^{r}$ in $\mathcal{D}$; (b) if $Q_{\rho}^{r}$ and $Q_{\sigma}^{s}$, in $\mathcal{D}$, are such that there is an inner point of $Q_{\rho}^{r}$ contained in $Q_{\sigma}^{s}$, then $Q_{\rho}^{\gamma} \subset Q_{\sigma}^{s}$. From (b), it follows that no two polyhedra in $\mathcal{D}$ can have an inner point in common unless they coincide.

$P^{n}$ and its boundary polyhedra $P_{\lambda}^{r}$ thus form a subdivision of $P^{n}$, which we call the canonical subdivision. If $\mathscr{D}$ is any subdivision of $P^{n}$, those polyhedra $Q_{\sigma}^{s}$ in $\mathcal{D}$ which are contained in a given polyhedron $Q_{\rho}^{r}$ in $\mathscr{D}$ form a subdivision of $Q_{\rho}^{r}$.

LemMa 7. If $Q^{r}$ is a polyhedron in a subdivision $\mathcal{D}$ of $P^{n}$, all inner points of $Q^{r}$ have the same type in $P^{n}$.

An inner point of $Q^{r}$ obviously has a type at least $r$ in $P^{n}$; hence the lemma is true for $r=n$; we prove it by induction, assuming it to hold for all $Q_{\sigma}^{s}$ in $\Phi$ with $s>r$. Let $\zeta$ be an inner point of $Q^{r}$; call $s$ its type in $P^{n}$, so that $s \geqq r$; $\zeta$ is then inner point of some $P_{\lambda}^{s}$; we need only show that all points of $Q^{r}$, sufficiently near to $\zeta$, are in $P_{\lambda}^{s}$. That will be the case if all points of $P_{\lambda}^{s}$, sufficiently near to $\zeta$, are in $Q^{r}$; for then, since $P_{\lambda}^{s}$ and $Q^{r}$ are of class at least $C^{1}$ and regular in $P^{n}$, we must have $s=r$, and $P_{\lambda}^{s}, Q^{r}$ must coincide in a neighborhood of point $\zeta$. If that is not so, then $\zeta$ must be a limiting point of inner points of $P_{\lambda}^{s}$ which are not in $Q^{r}$; as each of the latter points is an inner point of a polyhedron in $\mathcal{D}$, and there is only a finite number of such polyhedra, it follows that there is a $Q^{t}$ in $\mathcal{D}$, such that $\zeta$ is a limiting point of inner points of $Q^{t}$, each of which is an inner point of $P_{\lambda}^{s}$ and is not in $Q^{r}$. This implies that $\zeta \in Q^{t}$, and therefore $Q^{r} \subset Q^{t}$; hence $t>r$, as otherwise an inner point of $Q^{r}$ 
would be inner point of $Q^{t}$, and $Q^{r}$ would be the same as $Q^{t}$. By the induction assumption, the lemma holds for $Q^{t}$; as there are inner points of $Q^{t}$ which are inner points of $P_{\lambda}^{s}$, we have, therefore, $Q^{t} \subset P_{\lambda}^{s}$, and so $Q^{r} \subset P_{\lambda}^{s}$; this proves the lemma.

An immediate consequence is that all the polyhedra, in a subdivision $\mathscr{D}$ of $P^{n}$, which are contained in a given boundary polyhedron $P_{\lambda}^{r}$ of $P^{n}$, form a subdivision of that $P_{\lambda}^{r}$; this can be expressed by saying that every subdivision of $P^{n}$ is a refinement of the canonical subdivision. In particular, if a polyhedron $Q^{r}$, in a subdivision $D$ of $P^{n}$, contains at least one inner point of $P^{n}$, all inner points of $Q^{r}$ are inner points of $P^{n} ; Q^{r}$ is then called an inner polyhedron of the subdivision.

Lemma 8. $\mathcal{D}$ being a subdivision of $P^{n}$, and $z$ any point of $P^{n}$, the angles at $z$ of those polyhedra in $\mathcal{D}$ which contain $z$ form a subdivision of the angle of $P^{n}$ at $z$; the inner angles in the latter subdivision are the angles of the inner polyhedra in $\mathcal{D}$ which contain $z$.

In the proof of this lemma, we shall denote by $A(Q), Q$ being any regular subpolyhedron of $P^{n}$, the angle of $Q$ at $z$, if $z \in Q$, and the null-set otherwise. Let $x$ be any vector in $A\left(P^{n}\right)$, defined by an operator $X \phi=\lim \xi \cdot\left[\phi\left(z^{\prime}\right)-\phi(z)\right]$, where $z^{\prime}$ tends to $z$ within $P^{n}$ and $\xi$ tends to $+\infty$; as every $z^{\prime}$ is an inner point of a $Q_{\rho}^{\gamma}$ in $\mathcal{D}$, and there is only a finite number of such $Q_{\rho}^{\gamma}$, we may define $x$ by a sequence of $z^{\prime}$, all belonging to one and the same $Q_{\rho}^{r} ; A\left(Q_{\rho}^{r}\right)$ then contains $x$. Let $Q^{r}$ be a polyhedron of the lowest dimension in $\mathcal{D}$, such that $x \in A\left(Q^{r}\right)$; if $x$ were not an inner point of $A\left(Q^{r}\right)$, it would be in the angle at $z$ of a boundary polyhedron $Q^{\prime s}$ of $Q^{r}$, with $s<r$. The polyhedra in $D$ which are contained in $Q^{r}$ form a subdivision of $Q^{r}$, and so, by Lemma 7, those which are contained in $Q^{\prime s}$ form a subdivision of $Q^{\prime s} ; x$ would therefore be in the angle at $z$ of one of the latter polyhedra, which would be of dimension at most $s$, in contradiction with the definition of $Q^{r}$. This shows that $x$ is an inner vector of $A\left(Q^{r}\right)$. Suppose, that, at the same time, $x$ is an inner vector of $A\left(P^{n}\right)$; and let $x$ be defined by $X \phi=\lim \xi \cdot\left[\phi\left(z^{\prime}\right)-\phi(z)\right]$ where the $z^{\prime}$ are in $Q^{r}$; all $z^{\prime}$, sufficiently near to $z$, must be inner points of $P^{n}$ (otherwise $x$ would not be an inner point of $A\left(P^{n}\right)$ ), and so $Q^{r}$ must be an inner polyhedron of the subdivision $\mathscr{D}$. On the other hand, if $x$ is not an inner point of $A\left(P^{n}\right)$, it must be in the angle at $z$ of a boundary polyhedron $P_{\lambda}^{r}$ of $P^{n}$; since those polyhedra of $\mathcal{D}$ which are contained in $P_{\lambda}^{r}$ form a subdivision of $P_{\lambda}^{r}$, it follows, as above, that $x$ is then an inner point of an angle $A\left(Q^{s}\right)$, where $Q^{s}$ is a polyhedron in $\mathcal{D}$ and is contained in $P_{\lambda}^{r}$.

The proof of the lemma will now be complete if we show that, whenever $Q_{\rho}^{r}$ and $Q_{\sigma}^{s}$ belong to $\mathcal{D}$ and there is an inner point of $A\left(Q_{\rho}^{r}\right)$ contained in $A\left(Q_{\sigma}^{s}\right)$, $Q_{\rho}^{\sigma}$ itself is contained in $Q_{\sigma}^{s}$. Using induction, we may, in doing this, assume that the lemma is true for all subdivisions of polyhedra of dimension less than $n$ (the lemma is obviously true when $P^{n}$ has the dimension 1 ). The ques- 
tion being purely local, we need consider only a small neighborhood of $z$ in $P^{n}$, which we may identify with a convex angle in $R^{n}$; by the distance of two points in that neighborhood, we understand the Euclidean distance as measured in $R^{n}$. Let $Q^{r}$ be a polyhedron in $\mathcal{D}$, such that $z \in Q^{r}$; let $x$ be an inner vector of $A\left(Q^{r}\right)$, defined as above by an operator $X \phi=\lim \xi \cdot\left[\phi\left(z^{\prime}\right)-\phi(z)\right]$, where we may assume that $z^{\prime}$ runs over a sequence of inner points of $Q^{r}$ tending to $z$. In $R^{n}$, the direction of the vector $z z^{\prime}$ tends to that of the vector $x$. Our lemma will be proved if, assuming furthermore that $x$ is in the angle at $z$ of a polyhedron in $\mathcal{D}$ which does not contain $Q^{r}$, we show that this implies a contradiction. But the latter assumption implies that, if $w$ is a nearest point to $z^{\prime}$ in the union $W$ of those polyhedra in $\mathcal{D}$ which contain $z$ and do not contain $Q^{r}$, the direction of the vector $z w$ tends to that of $x$; we need therefore only show that this implies a contradiction.

$w$ must be contained in a polyhedron $Q_{\sigma}^{s}$ belonging to $\mathcal{D}$ and containing $Q^{r}$, since otherwise it could not be a nearest point to $z^{\prime}$ in $W$. Let $Q_{\mu}^{m}$ be the polyhedron in $\mathcal{D}$ of which $w$ is an inner point; this is contained in $Q_{\sigma}^{s}$, and cannot contain $Q^{r}$; it is therefore, by Lemma 7, contained in one of the boundary polyhedra $Q^{\prime t}$ of $Q_{\sigma}^{s}$. As there are only a finite number of possibilities for $Q_{\sigma}^{s}, Q_{\mu}^{m}, Q^{\prime t}$, we may, by replacing the sequence of points $z^{\prime}$ by a suitable subsequence, assume that these are the same for all $w$. We now identify a neighborhood of $z$ in $Q_{\sigma}^{s}$ with a convex angle in a Euclidean space $R^{s}$; as $z, z^{\prime}, w$, $Q^{r}, Q^{\prime t}$ are contained in $Q_{\sigma}^{s}$, we may, in the neighborhood of $z$, identify them with corresponding points and subsets of that convex angle, and $x$ with the corresponding vector in that same angle.

We have assumed that the direction of the vector $z w$ tends to that of $x$; therefore $Q^{r}$ cannot be the same as $Q_{\sigma}^{s}$, for $w$ is on the boundary of $Q_{\sigma}^{s}$, and $x$ is an inner vector of $Q^{r}$. Therefore $Q^{r}$ is contained in a boundary polyhedron $Q^{\prime \prime u}$ of $Q_{\sigma}^{s}$; the directions of the vectors $z z^{\prime}, z w$ tend to the direction of $x$; each point $w$ is in $Q^{\prime t}$, each point $z^{\prime}$ in $Q^{\prime \prime u}$, and, in the neighborhood of $z, Q^{\prime t}$ and $Q^{\prime \prime u}$ are the same as two boundary angles of the convex angle $Q_{\sigma}^{s}$; therefore $x$ must be in the angle at $z$ of $Q^{\prime t} \cap Q^{\prime \prime u}$, which, by Lemma 7 (applied to $\left.Q_{\sigma}^{s}\right)$, is the union of polyhedra of $\mathcal{D}$, so that $x$ is in the angle at $z$ of one of the latter polyhedra. Therefore (applying the induction assumption to $Q^{\prime \prime} u$ ) $Q^{r}$ is contained in that polyhedron, and a fortiori in $Q^{\prime t}$. Hence, applying the induction assumption to $Q^{\prime t}$, we get $Q^{r} \subset Q_{\mu}^{m}$, which contradicts an earlier statement.

We now define a cellular subdivision of a polyhedron $P^{n}$ as a subdivision $\mathcal{D}$. every polyhedron $Z_{\rho}^{r}$ in which is a differentiable cell (of the same class as $P^{n}$ ). The application of the results of $\$ 6$ to arbitrary polyhedra depends upon the following lemma:

LEMMA 9. Every differentiable polyhedron admits a cellular subdivision.

This is essentially contained in the work of S. S. Cairns on triangulation, 
and also in a subsequent paper of $\mathrm{H}$. Freudenthal on the same subject $\left({ }^{11}\right)$, and need not be proved here.

On a differentiable polyhedron, it is possible to define differentials and differential forms in the usual manner. Such a polyhedron will be called a Riemannian polyhedron if there has been given on it a positive-definite quadratic differential form, locally defined everywhere, in terms of local coordinates $z^{\mu}$, as $\sum_{\mu, \nu} g_{\mu \nu} d z^{\mu} d z^{\nu}$. We make once for all the assumption that our Riemannian polyhedra are of class at least $C^{3}$, and that the $g_{\mu \nu}$, which locally define their Riemannian structure, are of class at least $C^{2}$ wherever defined. If $P^{n}$ is such a polyhedron, and $Q^{p}$ any regular subpolyhedron of $P^{n}$, the Riemannian structure of $P^{n}$ induces again such a structure on $Q^{p}$; if $\zeta^{i}$ are local coordinates at a point $\zeta$ in $Q^{p}$, and the functions $z^{\mu}(\zeta)$ define the local imbedding of $Q^{p}$ in $P^{n}$ at that point, the structure of $Q^{p}$ at that point is defined by the form $\sum_{i, j} \gamma_{i j} d \zeta^{i} d \zeta^{i}$, where $\gamma_{i j}=\sum_{\mu, \nu} g_{\mu \nu} \partial z^{\mu} / \partial \zeta^{i} \cdot \partial z^{\nu} / \partial \zeta^{i}$. We shall denote by $d v(z)$ the intrinsic volume-element in $P^{n}$ at $z$, and by $d v(\zeta)$ the same in $Q^{p}$ at $\zeta$.

On a Riemannian polyhedron $P^{n}$, satisfying the above assumptions, we can define locally at every point $z$ the Riemannian curvature tensor, and hence, by formula (1) of $\S 1$, the invariant $\Psi(z)$. Let now $Q^{p}$ be a regular subpolyhedron of $P^{n}$, and $\zeta$ a point of $Q^{p}$; we shall denote by $N^{n-p}(\zeta)$ the normal linear manifold to $Q^{p}$ at $\zeta$, which is a submanifold of the tangent space to $P^{n}$ at $\zeta$. We denote by $\Gamma\left(Q^{p}, \zeta\right)$ the trace, on the unit-sphere, of the dual angle, taken in the tangent space to $P^{n}$, of the angle of $Q^{p}$ at $\zeta$. Furthermore, $x$ being any vector in $N^{n-p}(\zeta)$, we define $\Psi\left(\zeta, x \mid Q^{p}\right)$ by formulae (2), (3), (4), (5) of $\S 1$.

Let now $R^{s}$ be a polyhedron in a subdivision of $Q^{p}$. If $s=p=n$, we define $I\left(Q^{p}, R^{s}\right)$ as the integral of $\Psi(z) \cdot d v(z)$ over $R^{s}$. If $s<n$, we define $I\left(Q^{p}, R^{s}\right)$ as the integral of $\Psi\left(\zeta, \xi \mid R^{s}\right) d v(\zeta)$ when $\zeta$ describes the set of inner points of $R^{*}$ and $\xi$ describes, for each $\zeta$, the spherical cell $\Gamma\left(Q^{p}, \zeta\right)$. This implies that $I\left(Q^{p}, R^{s}\right)=0$ if the inner points of $R^{s}$ are of type greater than $s$ in $Q^{p}$, because $\Gamma\left(Q^{p}, \zeta\right)$ has then a dimension less than $n-s-1$. If, therefore, we consider the sum $\sum_{s, \sigma} I\left(Q^{p}, R_{\sigma}^{s}\right)$, taken over all polyhedra $R_{\sigma}^{s}$ of a subdivision of $Q^{p}$, this sum has the same value as the similar sum taken for the canonical subdivision of $Q^{p}$; the value of that sum is therefore independent of the subdivision by means of which it is defined, and we may write:

$$
\sigma\left(Q^{p}\right)=\sum_{s=0}^{p} \sum_{\sigma} I\left(Q^{p}, R_{\sigma}^{s}\right),
$$

the sum being taken over all polyhedra of any subdivision of $Q^{p}$.

(11) See S. S. Cairns' expository paper, Triangulated manifolds and differentiable manifolds, in Lectures in topology, University of Michigan Conference of 1940, University of Michigan Press, 1941, p. 143, where references will be found to Cairns', Freudenthal's and Whitehead's publications. 
$\Psi(z)$ has been defined by using $P^{n}$ as the underlying Riemannian space. If, on the other hand, we use $Q^{p}$ as underlying space, we may, substituting $p$ for $n$ in formula (1) of $\$ 1$ and using the metric and curvature tensors of $Q^{p}$, define the similar invariant for $Q^{p}$, which we denote by $\Psi_{0}(\zeta)$. Similarly, $\xi_{0}$ being a normal unit-vector to $R^{\boldsymbol{s}}$ in the tangent space to $Q^{p}$ at a point $\zeta$ of $R^{\boldsymbol{s}}$, we define $\Psi_{0}\left(\zeta, \xi_{0} \mid R^{s}\right)$ by the formulae, similar to (2)-(5) of $\S 1$, where $Q^{p}$ is taken as underlying space instead of $P^{n}$. We also define $\Gamma_{0}\left(Q^{p}, \zeta\right)$ as the trace, on the unit-sphere, of the dual angle, taken in the tangent space to $Q^{p}$, of the angle of $Q^{p}$ at $\zeta$. And we define $I_{0}\left(Q^{p}, R^{s}\right)$ as the integral of $\Psi_{0}(\zeta) d v(\zeta)$ over $R^{s}$ if $s=p$, and, if $s<p$, as the integral of $\Psi_{0}\left(\zeta, \xi_{0} \mid R^{s}\right) d v(\zeta)$ when $\zeta$ describes the set of inner points of $R^{s}$ and $\xi_{0}$ describes, for each $\zeta$, the spherical cell $\Gamma_{0}\left(Q^{p}, \zeta\right)$. By the same argument as above, we see, that the sum

$$
\sigma_{0}\left(Q^{p}\right)=\sum_{s, \sigma} I_{0}\left(Q^{p}, R_{\sigma}^{s}\right),
$$

taken over all polyhedra $R_{\sigma}^{s}$ of a subdivision of $Q^{p}$, is independent of that subdivision. This sum, taken for the canonical subdivision of $Q^{p}$, is the same (except for slight changes of notations) as the sum that occurs in the righthand side of the formula in Theorem II of $\S 1$, when that theorem is applied to $Q^{p}$. With our present notations, we may, therefore, re-state our Theorem II in the following terms:

TheOREM II. For every Riemannian polyhedron $Q^{p}, \sigma_{0}\left(Q^{p}\right)=(-1)^{p} \cdot \chi^{\prime}\left(Q^{p}\right)$.

We shall first prove that $\sigma\left(Q^{p}\right)=\sigma_{0}\left(Q^{p}\right)$. As $\sigma\left(Q^{p}\right), \sigma_{0}\left(Q^{p}\right)$ can be defined from the canonical subdivision of $Q^{p}$, it will be enough to prove that, for every $Q_{\rho}^{r}$ in that subdivision (that is, either $Q^{p}$ or one of its boundary polyhedra), $I\left(Q^{p}, Q_{\rho}^{r}\right)=I_{0}\left(Q^{p}, Q_{\rho}^{r}\right)$; and this will be proved if we prove that

$$
\Psi_{0}(\zeta)=\int_{\Gamma\left(Q^{p}, \zeta\right)} \Psi\left(\zeta, \xi \mid Q^{p}\right)
$$

whenever $\zeta$ is an inner point of $Q^{p}$, and

$$
\int_{\Gamma_{0}\left(Q^{r}, \zeta\right)} \Psi_{0}\left(\zeta, \xi_{0} \mid Q^{r}\right)=\int_{\Gamma_{\left(Q^{r}, \zeta\right)}} \Psi\left(\zeta, \xi \mid Q^{r}\right)
$$

whenever $\zeta$ is an inner point of a boundary polyhedron $Q^{r}$ of $Q^{p}$. But these identities have been proved, as formulae (6) and (7) of $\$ 6$, in the particular case when $P^{n}$ is a Riemannian cell; they are purely local, and depend only upon the angle of $Q^{p}$ at $\zeta$, the $g_{\mu \nu}$ and their first and second derivatives and the first and second derivatives of the $z^{\mu}(\zeta)$ at that point; hence they hold in general.

We now prove the important additivity property of the function $\sigma\left(Q^{p}\right)$ $=\sigma_{0}\left(Q^{p}\right)$ : 
LEMma 10. For any subdivision of $P^{n}$, the formula holds:

$$
(-1)^{n} \sigma\left(P^{n}\right)=\sum_{r, \rho}^{\prime}(-1)^{r} \sigma\left(Q_{p}^{r}\right)
$$

where $\sum$ ' denotes summation over all inner polyhedra $Q_{p}^{r}$ of the subdivision.

Call $S$ the sum on the right-hand side. Replacing the $\sigma\left(Q_{\rho}^{r}\right)$ by their definition, we see that

$$
S=\sum_{r, \rho, s, \sigma} \epsilon_{r, \rho, 8, \sigma} I\left(Q_{\rho}^{r}, Q_{\sigma}^{\prime}\right),
$$

where the sum is taken over all values of $r, \rho, s, \sigma$, and $\epsilon_{r, \rho, s, \sigma}$ has the value $(-1)^{r}$ whenever $Q_{\rho}^{r} \supset Q_{\sigma}^{s}$ and $Q_{\rho}^{r}$ is an inner polyhedron of the subdivision, and the value 0 otherwise. We may write, therefore:

where $J_{s, \sigma}$ is defined by

$$
S=\sum_{s, \sigma} J_{s, \sigma}
$$

$$
J_{s, \sigma}=\sum_{r, \rho} \epsilon_{r, p, s, \sigma} I\left(Q_{\rho}^{r}, Q_{\sigma}^{\prime}\right) ;
$$

the latter sum may be restricted to those values of $r, \rho$ for which $Q_{\rho}^{r}$ contains $Q_{p}^{s}$ and is an inner polyhedron.

We first calculate $J_{s, \sigma}$ in the case $s=n$; the sum then contains only one term, and we have:

$$
J_{n, \sigma}=(-1)^{n} I\left(Q_{\sigma}^{n}, Q_{\sigma}^{n}\right)=(-1)^{n} I\left(P^{n}, Q_{\sigma}^{n}\right) .
$$

We now take the case $s<n$. From the definition of $I\left(Q_{\rho}^{r}, Q_{\sigma}^{s}\right)$, it follows that $J_{s, \sigma}$ is the integral of $\Psi\left(\zeta, \xi \mid Q_{\sigma}^{s}\right) d v(\zeta)$ when $\zeta$ describes the set of inner points of $Q_{\sigma}^{s}$, and the integration in $\xi$, for each $\zeta$, is over the chain:

$$
\Delta=\sum_{r, \rho} \epsilon_{r, \rho, s, \sigma} \Gamma\left(Q_{p}^{r}, \zeta\right) .
$$

Now $\Gamma\left(Q_{\rho}^{r}, \zeta\right)$, as a chain on $S^{n-8-1}$, is the same as the outer angle, taken in $N^{n-s}(\zeta)$ according to our definitions in $\S 2$, of the trace on $N^{n-s}(\zeta)$ of the angle of $Q_{\rho}^{r}$ at $\zeta$. In the sum for $\Delta$, we have all those $Q_{\rho}^{r}$ which are inner polyhedra of the subdivision and which contain $Q_{\sigma}^{s}$, that is, which contain $\zeta$ (since $\zeta$ is an inner point of $Q_{\sigma}^{s}$ ); by Lemma 8, their angles at $\zeta$ are the inner angles of a subdivision of the angle of $P^{n}$ at $\zeta$; since all those angles contain the tangent manifold to $Q_{\sigma}^{s}$ at $\zeta$, their traces on $N^{n-\cdot}(\zeta)$ bear the same relationship to the trace on $N^{n-s}(\zeta)$ of the angle of $P^{n}$; we may therefore apply to the outer angles of those traces Theorem III of $\S 2$, which gives here $\Delta=(-1)^{n} \Gamma\left(P^{n}, \zeta\right)$, and therefore:

$$
J_{s, \sigma}=(-1)^{n} I\left(P^{n}, Q_{\sigma}^{*}\right),
$$

which proves the lemma. 
Lemma 10 shows that if Theorem II holds for every cell in a certain cellular subdivision of $P^{n}$, it holds for $P^{n}$; for, if all $Q_{\rho}^{r}$ are cells and Theorem II holds for them, we have $\sigma\left(Q_{\rho}^{r}\right)=(-1)^{r} \chi^{\prime}\left(Q_{\rho}^{r}\right)=1$, and the right-hand side of the formula in Lemma 10 reduces to the inner characteristic of $P^{n}$, as calculated from the given subdivision. Since every polyhedron admits a cellular subdivision, it will now be enough to prove Theorem II for cells. By $\S 6$, we know it to hold for an "imbeddable" cell $K^{n}$, that is, for one in which the $g_{\mu \nu}$ are defined, as in $\S 6$, by a mapping $f$ of $K^{n}$ into a Euclidean space $R^{N}$.

We next take the case of an analytic cell, which we may define by taking a convex cell $K^{n}$, and $n(n+1) / 2$ functions $g_{\mu \nu}(z)$, analytic over $K^{n}$, such that the quadratic form with the coefficients $g_{\mu \nu}(z)$ is positive-definite for every $z$ in $K^{n}$. By Cartan's theorem( $\left.{ }^{12}\right)$, every point of $K^{n}$ has a neighborhood which can be analytically and isometrically imbedded in a Euclidean space. If, therefore, we subdivide $K^{n}$ into sufficiently small convex cells (for example, by parallel planes), the Riemannian structure induced on any one of the latter by the given structure in $K^{n}$ can be defined by an analytic mapping into some Euclidean space, and therefore the results of $\$ 6$ apply to all cells in that subdivision. Therefore Theorem II holds for $K^{n}$.

We now take an arbitrary cell, defined as above by a convex cell $K^{n}$ and functions $g_{\mu \nu}(z)$ over $K^{n}$, the latter being only assumed to be of class $C^{2}$; by a theorem of $\mathrm{H}$. Whitney $\left({ }^{13}\right)$, the $g_{\mu \nu}(z)$ can be uniformly approximated, together with their first and second derivatives, by analytic functions and their derivatives. But the expression $\sigma\left(K^{n}\right)$, considered (for a given $K^{n}$ ) in its dependence upon the $g_{\mu \nu}$, depends continuously upon the $g_{\mu \nu}$ and their first and second derivatives; for the integrands $\Psi$ are rational expressions in the $g_{\mu \nu}$, their first and second derivatives, and the components $\xi_{\mu}$ of vector $\xi$; the denominators in the $\Psi$ consist merely of the determinants $g, \gamma$, which are bounded away from $0 ; d v(z)$ is $g^{1 / 2} \cdot d z, d v(\zeta)$ is $\gamma^{1 / 2} \cdot d \zeta$. As to $\xi$, we may put $\xi_{\mu}=\omega \cdot \bar{\xi}_{\mu}$, where $\bar{\xi}_{\mu}$ describes the trace of the dual of the angle of $K^{n}$ at $\zeta$ on the surface $\sum_{\mu}\left(\bar{\xi}_{\mu}\right)^{2}=1$, which is independent of the $g_{\mu \nu}$, and $\omega=\left(\sum_{\mu \nu} g^{\mu \nu} \cdot \bar{\xi}_{\mu} \bar{\xi}_{\nu}\right)^{-1 / 2}$. Expressing $\xi_{\mu}, d \xi$ in terms of the $\bar{\xi}_{\mu}$, we get expressions which are continuous in the $g_{\mu \nu}$. Since $\sigma\left(K^{n}\right)$ is equal to 1 whenever the $g_{\mu \nu}$ are analytic, it follows that it is always 1 , and this completes our proof.

Our main result is thus proved in full. Owing, however, to the very unsatisfactory condition in which the theory of differentiable polyhedra has remained until now, the scope of our Theorem II may not be quite adequate for some applications, and we shall add a few remarks which properly belong

(12) Loc. cit. Footnote 4.

(13) H. Whitney, Analytic extensions of differentiable functions defined in closed sets, Trans. Amer. Math. Soc. vol. 36 (1934) p. 63 (see Lemma 2, p. 69 and Lemma 5, p. 74; as to the latter lemma, which is due to L. Tonelli, cf. C. de la Vallee Poussin, Cours d'analyse infinitésimale, vol. 2, 2d edition, Louvain-Paris, 1912, pp. 133-135). 
to that theory (to which part of this section may also be regarded as a contribution).

One would feel tempted to regard as a differentiable polyhedron any compact subset of a differentiable manifold which can be defined by a finite number of inequalities $\phi_{\nu}(z) \geqq a_{\nu}$, where the $\phi_{\nu}$ are functions of the same class $C^{m}$ as the manifold; and one would wish to be able to apply the Gauss-Bonnet formula to such sets.

Now, a compact set $P$ determined, on a manifold $M^{n}$ of class $C^{m}$, by inequalities $\phi_{\nu}(z) \geqq a_{\nu}$, the $\phi_{\nu}$ being functions of class $C^{m}$ in finite number, actually is a differentiable polyhedron of class $C^{m}$, according to our definitions, if the following condition is fulfilled: (A) For any subset $S$ of the set of indices $\nu$, consisting of $s$ elements, and any point $z$ of $M^{n}$ satisfying $\phi_{\sigma}(z)=a_{\sigma}$ for $\sigma \in S$ and $\phi_{\nu}(z)>a_{\nu}$ for $\nu \notin S$, the matrix $\left\|\partial \phi_{\sigma} / \partial z^{\mu}\right\|$ (where $\sigma$ runs over $S$ and $\mu$ ranges from 1 to $n$ ) is of rank $s$. In fact, if condition (A) is fulfilled, let $z$ be any point of $P$; call $S$ the set of all those indices $\sigma$ for which $\phi_{\sigma}(z)=a_{\sigma}$; by condition (A), their number $s$ is at most $n$, and we may take the $\phi_{\sigma}(z)$ as $s$ of the local coordinates at $z$; the neighborhood of $z$ in $P$ is then an image of class $C^{m}$ of the angle determined in $R^{n}$ by the $s$ inequalities $x_{\sigma} \geqq 0$.

If condition (A) is not satisfied, $P$ need not be a differentiable polyhedron, and indeed it can be shown by examples that "pathological" circumstances

- may occur. It can be shown, however, that condition (A) is fulfilled, in a suitable sense, for "almost all" values of the $a_{\nu}$, when the $\phi_{\nu}$ are given. This gives the possibility of extending the validity of Theorem II to cases when (A) is not fulfilled, by applying it to suitable neighboring values of the $a_{\nu}$ and passing to the limit. Alternatively, almost any "reasonable" definition of a differentiable polyhedron, more general than ours, will be found to be such that our proofs of Lemmas 7 and 8 will remain valid; all our further deductions will then hold provided triangulation is possible.

Finally, it may also be observed that the set $P$, defined as above by inequalities $\phi_{\nu}(z) \geqq 0$, can be considered as a limiting case of the set $P_{c}^{n}$ defined by the inequalities $\phi_{\nu}(z) \geqq 0, \prod_{\nu} \phi_{\nu}(z) \geqq \epsilon$, where $\epsilon$ is any number greater than 0 . The latter is a polyhedron with a single boundary polyhedron $P_{e}^{n-1}$ which is a compact manifold of dimension $n-1$; it may be considered as derived from $P$ by "rounding off the edges." We may therefore apply Theorem II to $P_{e}^{n}$; and it is to be expected that the formula thus obtained will tend to a formula of the desired type when $\epsilon$ tends to 0 . In fact, this idea could probably be used in order to derive our main theorem from the special case of polyhedra $P^{n}$ bounded by a single $(n-1)$-dimensional manifold.

Haverford College, Haverford, Pa. 